\title{
Tree Encoding in the ITU-T G.711.1 Speech Coder
}

\author{
Abdul Hannan Khan
}

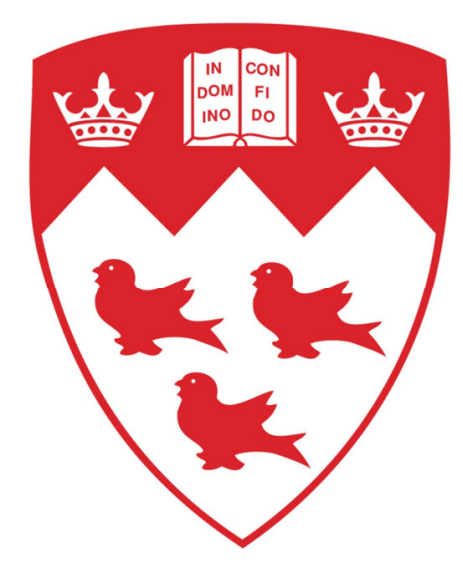

Department of Electrical Computer and Software Engineering McGill University

Montreal, Canada

November, 2010

A thesis submitted to McGill University in partial fulfillment of the requirements for the degree of Master of Engineering

(C) 2010 Abdul Hannan Khan 


\section{Tree Encoding in the ITU-T G.711.1 Speech $\quad 2010$ Coder}

\section{ABSTRACT}

This thesis examines further enhancement to ITU-T G.711.1 speech coder. The original G.711 coder is effectively a low band $\mu$-law quantizer. The G.711.1 extension adds noise feed-back and lower band enhancement layer apart from the higher-band. To further improve the core lower-band coding performance the use of both vector quantization and delayed decision multi-path tree encoder in the above coder at the low band portion is studied. The delayed decision multi-path tree encoding is implemented by the $(M, L)$ - algorithm. The new quantizer takes into account past history, and hence, the error propagation due to noise feed-back, and codes multiple samples under $\mu$-law. The final bitstream is compatible with the G.711.1 decoder and, hence, with the original G.711 decoder. An evaluation method, ITU-T P.862 perceptual evaluation of speech quality (PESQ), is used to evaluate the performance. Both the vector quantizer and tree encoder have better performance than the original core layer encoder in terms of perceptual quality, though they are limited by the increased computational complexity. Future studies are suggested. 


\section{Coder}

\section{SOMMAIRE}

Cette thèse étudie en détail les améliorations apportées au codeur de la parole ITU-T G.711.1. Le codeur original G.711 est en fait un quantificateur $\mu$-law. Le prolongement large-bande G.711.1 utilise le façonnage du bruit ainsi qu'une couche d'amélioration de la bande-basse en plus de la bande-haute. Afin d'améliorer le codage de la bande-basse principale, nous étudions l'utilisation de quantification vectorielle et la décision à retardement. Le codeur arboriforme avec décision à retardée est réalisé par l'algorithme $(M, L)$. Le nouveau quantificateur considère l'information passée et par conséquent, il considère également la propagation de l'erreur engendrée par le façonnage du bruit. Il code plusieurs échantillons par $\mu$ law. Le flot binaire final est compatible avec le décodeur du prolongement largebande G.711.1 et donc naturellement avec le décodeur du G.711 original. Une méthode d'évaluation, ITU-T P.862 (PESQ) est utilisée pour évaluer la performance. Les résultats montrent que la quantification vectorielle et le codeur arboriforme sont perceptuellement plus performants que le codeur original de la bande principale. Nous notons tout de même qu'ils sont numériquement plus complexes à réaliser. Des études supplémentaires sont suggérées. 


\section{ACKNOWLEDGEMENTS}

I would like to thank Dr. Peter Kabal for his continued guidance, supervision, friendliness and wise counsel throughout the course of this study. I'm grateful to my family, especially my parents, for their continuous encouragement and support. Also I thank Mr. Mohamed Konate for translating the abstract of this thesis into French. Finally, I would like to thank McGill University and its staff for all the resources provided that were used during the period of this study. 


\section{Coder}

\section{TABLE OF CONTENTS}

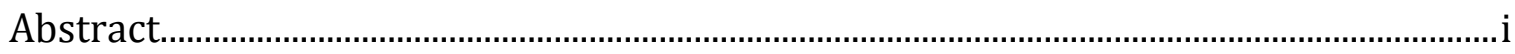

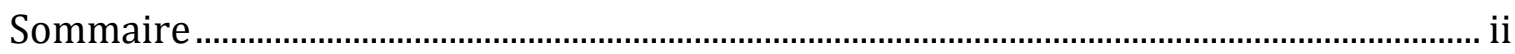

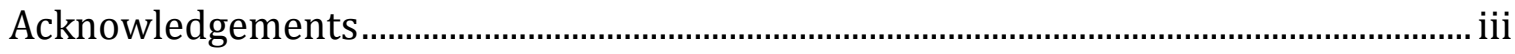

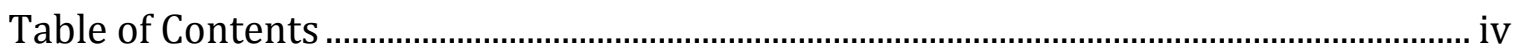

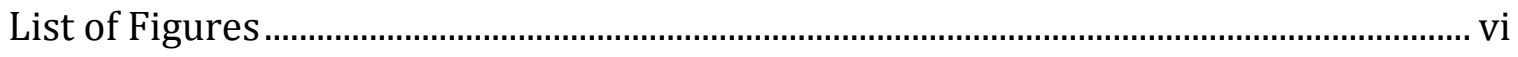

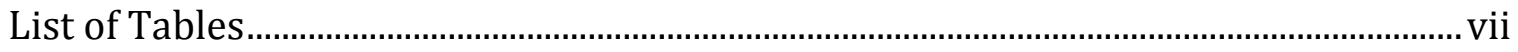

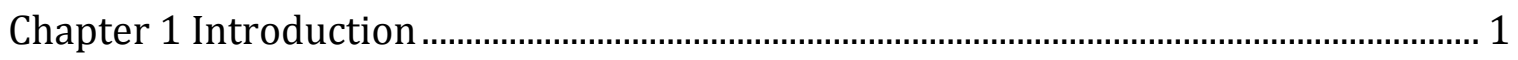

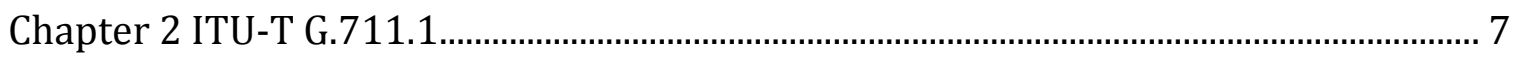

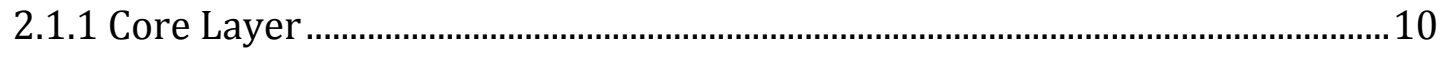

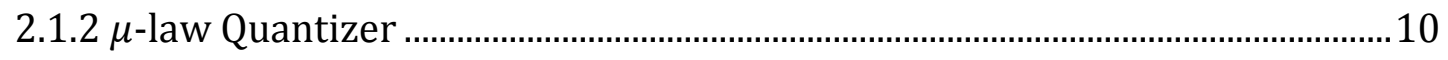

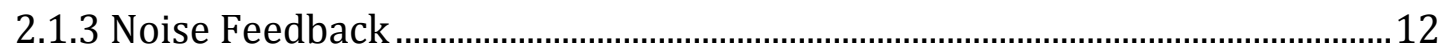

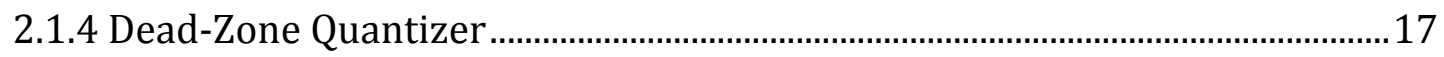

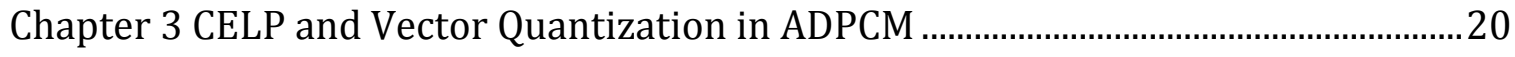

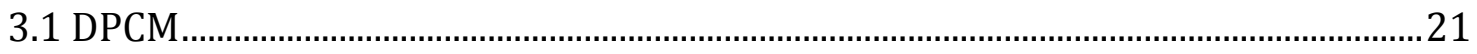

3.2 ADPCM

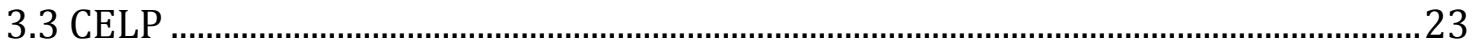

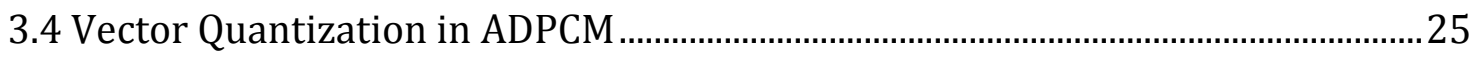

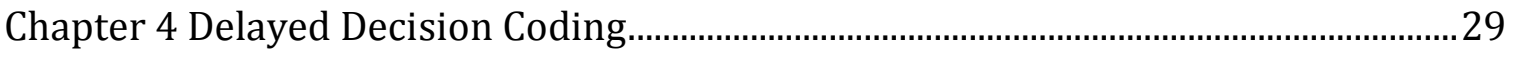




\section{Coder}

4.1 Tree Encoding.

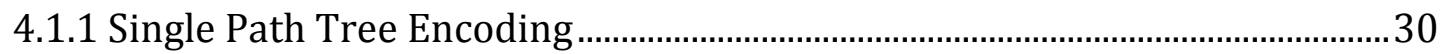

4.1.2 Multi-Path Tree Encoding: The $(M, L)$-Algorithm .............................................. 32

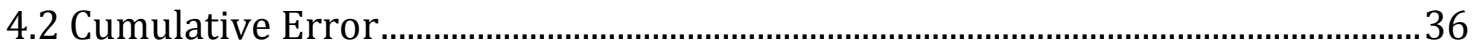

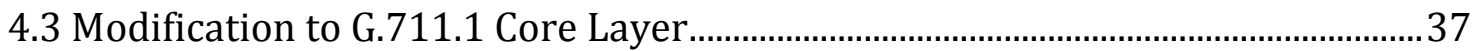

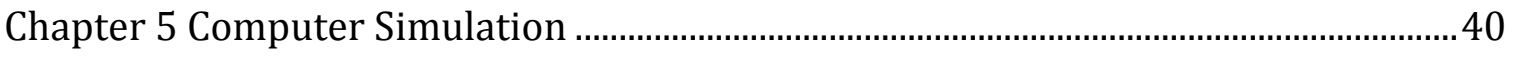

5.1 Sub Optimal Approach to Reduce Complexity .......................................................... 40

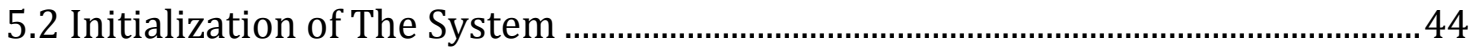

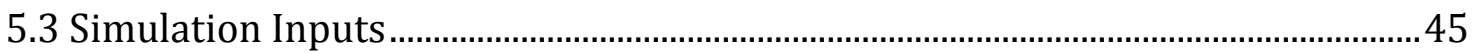

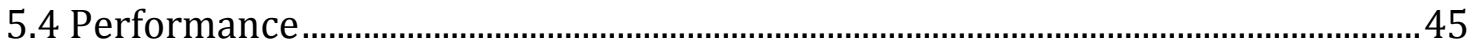

5.4.1 Perceptual Evaluation of Speech Quality............................................................. 45

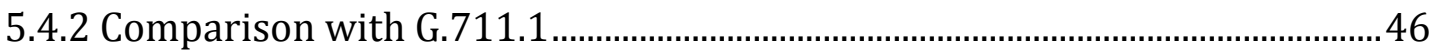

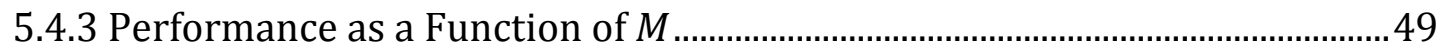

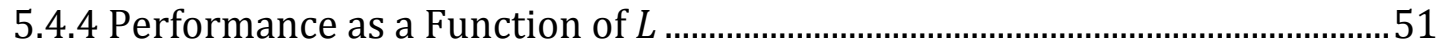

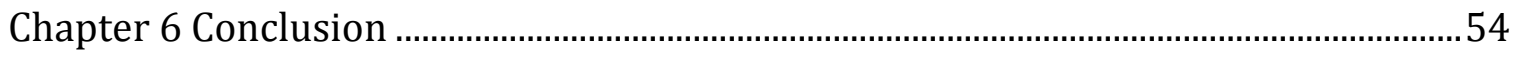

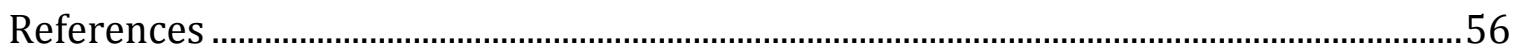




\section{Coder}

\section{LIST OF FIGURES}

Figure 2-1 - Block diagram of G.711.1 encoder

Figure 2-2 - Lower-band encoder 10

Figure 2-3 - Noise shaping

Figure 2-4 - Quantization noise without noise feedback (left) and with noise feedback (right) [4]

Figure 2-5 - Quantization noise without noise feedback (left) and with noise feedback (right) [2]

Figure 3-1 - DPCM Coding; encoder on the left, decoder on the right. 21

Figure 3-2 - ADPCM encoder block diagram

Figure 3-3 - CELP Encoder.

Figure 3-4 - Rearranged ADPCM encoder structure to show noise feedback. .25

Figure 3-5 - VQ in ADPCM encoder with noise feedback. 26

Figure 3-6 - VQ in ADPCM encoder with noise feedback - form 1...............................28

Figure 3-7 - VQ in ADPCM encoder with noise feedback - form 2................................28

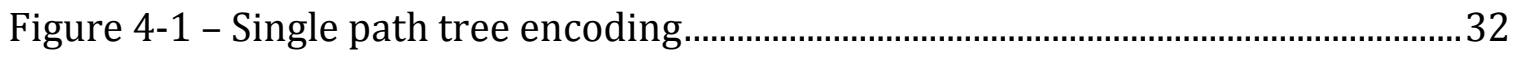

Figure 4-2 - Multi-path tree encoding

Figure 4-3 - G.711.1 core layer with codebook VQ 38

Figure 4-4 - G.711.1 core layer with codebook VQ - rearranged.

Figure 5-1 - PESQ score of tree encoding as a function of $\mathrm{M}$, with $\mathrm{L}=6$ at $-40 \mathrm{~dB}$. For the first point $M=1$ and $L=1$. The performance of $G .711 .1$ core layer is provided for comparison 


\section{Coder}

Figure 5-2 - PESQ score of tree encoding as a function of $\mathrm{L}$, with $\mathrm{M}=6$ at $-40 \mathrm{~dB}$. For the first point $\mathrm{L}=1$ and $\mathrm{M}=1$. The performance of $\mathrm{G} .711 .1$ core layer is provided for

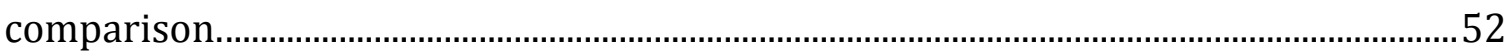

\section{LIST OF TABLES}

Table 5-1 - Multiplication and addition operations per sample of different G.711.1

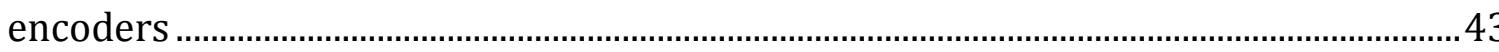

Table 5-2 - Comparison of different G.711.1 encoders using PESQ ................................47 


\section{Chapter 1 InTRODUCTION}

Speech coding is the process by which an analog speech signal, continuous in both time and amplitude, is digitized, i.e. converted to a speech signal discrete in both time and amplitude. The signal in the process is compressed, hence, taking fewer resources for storage and/or transmission. Speech coding has some differences with audio coding. More established models are available for speech as compared to other audio signals. Psychoacoustics also plays its role in speech coding. Speech is coded and transmitted such that only information relevant to the human auditory system is transmitted. Higher quality at a lower bit rate can be further achieved by making use of signal redundancy and masking the distortions created by coding such that they become imperceptible. Even a narrow band $(<4,000 \mathrm{~Hz})$ signal is enough for intelligibility. It needs to be clarified that intelligibility is different from pleasantness. Understanding of the content, speaker identity, timbre and tone are all vital for the former. Pleasantness is about whether the degraded speech signal is subjectively irritating or not. 


\section{Tree Encoding in the ITU-T G.711.1 Speech Coder}

The immediate advantage of speech coding comes in the form of reduced data storage capacity required. High quality speech can now be stored on a physical media without consumption of a lot of memory space. Once speech is coded it can be transmitted as data, utilizing the same public switched loop circuits. Voice and data signals can be sent on the same channel. Digital speech signals allow better security. They can be encrypted and/or scrambled with greater efficiency. High quality at low bit rates have made it possible to meet growing demands of wireless communication. Today high quality speech coding is available at $8 \mathrm{kbps}$, although this thesis deals with a speech coder working at $64 \mathrm{kbps}$ or more.

There are different parameters of speech coder performance. The aim of a speech coder is to improve the speech quality while reducing the bit rate, communication delay and complexity. The five-point scale on which speech quality is mostly evaluated is known as the mean-opinion score (MOS) scale. It is a subjective test and is averaged over a large set of data, speakers and listeners. Scores of 3.5 or higher are generally considered to have good levels of intelligibility. Another similar scale based on comparison of the original and degenerated signal is the perceptual evaluation of sound quality (PESQ). PESQ is an objective measure of sound quality. Hence, the requirement of having a large set of listeners is eliminated while the scale is similar. There will always be a slight communication delay as speech coders have to process data, and they often work in blocks of samples. The constraint on communication delay is application dependent. Even in real time communication it varies from 1 to $500 \mathrm{~ms}$; higher delays are permissible in video 


\section{Tree Encoding in the ITU-T G.711.1 Speech Coder}

telephony. Complexity is measured in terms of number of arithmetic operations performed and memory requirements. Higher complexity often results in higher communication delays and in higher power consumption. With advancements in chip design technology higher complexity speech coders can now be implemented with acceptable delays and power consumption.

Generally speech coders are divided into three classes; waveform coders, source coders and hybrid coders. Waveform coders are the simplest to implement, from a complexity point of view. They are largely independent of the input signal and try to reconstruct a signal whose waveform is as close to the input. For a time domain coding approach the simplest coder involves sampling and quantizing the input signal. One coder who works on this principle is the pulse code modulation (PCM) coder. Logarithmic quantization is used to provide same quality of reconstruction at a reduced bit rate. Such a coder has a bit rate of $64 \mathrm{kbps}$. Another example of a waveform coder is the differential pulse code modulation (DPCM) coder. The difference between the input signal and the predicted signal is coded. This reduces the number of bits required for coding. A typical bit rate for such a coder is $32 \mathrm{kbps}$. In frequency domain waveform coding, a signal is divided up into different bands and each is coded and transmitted individually. Examples of such frequency domain waveform coding are sub-band coding (SBC) and adaptive transform coding (ATC). These coding techniques are a bit more complex than time domain coding techniques because of the filtering required to split the input signal into sub-bands. 


\section{Tree Encoding in the ITU-T G.711.1 Speech Coder}

Sources coders are typically the lower bit rate coders. Source coders try to model the source of the input signal. The parameters of the source model are then transmitted. A time-varying filter is used to model the vocal tract. The excitation signal depends on whether the input is voiced or unvoiced speech. In the case of the former a train of pulses is used while for the latter white noise is used. The period of the pulses is the same as the pitch period of the voiced speech. Filter coefficients, gain factors, voiced/unvoiced speech decision and pitch period are the parameters transmitted. There is usually a loss of naturalness in the reconstructed speech from a source coder. The reconstructed speech has a synthetic feel but this may be acceptable where low bit rate is preferred over naturalness of speech. Linear predictive coding (LPC) coder is an example of such a source coder. It operates around $2.4 \mathrm{kbps}$.

Hybrid coders, as the name suggests, tend to find a compromise between waveform coders and sources coders, both in terms of how they code the signal and the bit rate. One of the most important hybrid coders is the code excited linear predictive (CELP) coder. It is an analysis-by-synthesis coder. It employs linear prediction and then quantizes the residual signal. The parameters of the linear prediction filter and the quantized residual signal are transmitted. The residual signal is used to excite the synthesis filter in the receiver. The quantization of the residual signal is such that to minimize the error and match the input signal as closely as possible. Operating between 4.8 and $16 \mathrm{kbps}$, these coders produce good quality reconstructed speech. 


\section{Tree Encoding in the ITU-T G.711.1 Speech Coder}

This thesis presents work done on a speech coder. ITU-T standard G.711.1 is a wideband embedded extension to G.711 PCM encoded speech [2]. The extension was approved in March 2008. The G.711.1 wideband extension adds noise feedback and a lower-band enhancement layer, as well as a high band encoding layer. The noise feedback tries to perceptually mask the quantization noise introduced by the PCM quantizer. The perceptual filter is based on the linear prediction filter. What the enhancement layer does is that it allows more bits to be used for encoding, hence, increasing the number of quantization levels. This reduces the quantization noise at the expense of more bits. The higher band encoding is based on modified discrete cosine transform (MDCT) and uses an interleave conjugate-structure vector quantizer (CSVQ). This thesis will be talking about the lower-band.

This research studies the effect on G.711.1 speech coder by incorporating vector quantization (VQ) and delayed decision multi-path tree encoding. While G.711.1 is concerned with both low and high bands, this thesis concerns only with the low band. The delayed decision multi-path tree encoding is implemented by the $(M, L)$-algorithm as suggested in [3]. $M$ is the maximum number of tree paths available after quantizing a block of input samples and $L$ is the maximum depth of the tree. $L$ also dictates the delay after which an input block is coded. Because the noise feedback filter has memory, a decision made at a certain instance has effect on decisions made in the future. The new quantizer takes into account past history (or future values, depending on how you look at it ), and hence, the error propagation due to noise feedback is taken into consideration as well when making the final 


\section{Tree Encoding in the ITU-T G.711.1 Speech Coder}

decision on the code. One major advantage is that the final bit-stream is compatible with the G.711.1 decoder.

The working of the G711.1 speech coder is studied in Chapter 2. The lowerband quantizer and the noise feedback filter are discussed in detail as these are common to the new coder; the delayed decision multi-path tree encoding is implemented in the lower-band. Chapter 3 deals with CELP and adaptive differential pulse code modulation (ADPCM), as it is from there that the idea of using vector quantization in G.711.1 originated. Chapter 4 describes delayed decision coding, multi-path tree encoding to be precise, in detail. Simulation results are provided in Chapter 5. With Chapter 6 this thesis is concluded. 


\section{Tree Encoding in the ITU-T G.711.1 Speech 2010 Coder}

\section{Chapter 2 ITU-T G.711.1}

ITU-T G.711.1's predecessor, G.711, uses PCM with logarithmic quantization. With a logarithmic scale, 12 bits of resolution can be achieved by using only 8 bits per sample. Two such scales exist, $A$-law and $\mu$-law. Except for slight differences in quantization levels both are essentially the same. In this thesis $\mu$-law has been used and all further mention should be taken as such unless stated otherwise. These algorithms provide good quality speech coding at very low complexity while saving $33 \%$ bandwidth as compared to linear quantization. These properties found them use in digital telephony and have not been replaced. In 2008 ITU-T recommended a wideband extension to G.711, ITU-T G.711.1 wideband embedded extension for PCM [2]. The new coder has an embedded structure and is backward compatible with existing G.711 coders.

The conventional G.711 log companded PCM encoder has bandwidth of 300-3400 $\mathrm{Hz}$ at $64 \mathrm{kbps}$, and takes input sampled at $8 \mathrm{kHz}$. In G.711.1 all these values have been increased. For input sampled at $16 \mathrm{kHz}$ it has a bandwidth of $50-7000 \mathrm{~Hz}$ at 80 and $96 \mathrm{kbps}$, while for signal sampled at $8 \mathrm{kHz}$ it has a bandwidth of $0-4000 \mathrm{~Hz}$ 


\section{Tree Encoding in the ITU-T G.711.1 Speech Coder}

at 64 and $80 \mathrm{kbps}$. Different bit rates are available because of the embedded structure. The new standard has three layers:

- Core layer (Layer 0): always present at $64 \mathrm{kbps}$

- Lower-band enhancement layer (Layer 1): optional with addition of $16 \mathrm{kbps}$

- Higher-band layer (Layer 2): optional with addition of $16 \mathrm{kbps}$

The core layer, at $64 \mathrm{kbps}$, is compatible with G.711 decoder. Different combination of these three layers gives rise to four different encoding modes.

- R1: only core layer at a sampling rate of $8 \mathrm{kHz}$ and bit rate of $64 \mathrm{kbps}$

- R2a: core layer and lower-band enhancement layer at a sampling rate of $8 \mathrm{kHz}$ and bit rate of $80 \mathrm{kbps}$

- R2b: core layer and higher-band layer at a sampling rate of $16 \mathrm{kHz}$ and bit rate of $80 \mathrm{kbps}$

- R3: all three layers at a sampling rate of $16 \mathrm{kHz}$ and bit rate of $96 \mathrm{kbps}$

Figure 2-1 gives a higher level look at the G.711.1 encoder. The wideband input signal sampled at $16 \mathrm{kHz}$ is split by a 32-tap quadrature mirror filterbank (QMF). The lower-band encoding produces two streams; the G.711 compatible core layer and the lower-band enhancement layer. MDCT is applied to the higher-band signal and the frequency domain coefficients are encoded by a CSVQ. The final bitstream is a multiplexed version of all three. In the case of $8 \mathrm{kHz}$ sampled input signal the QMF is by-passed and the signal fed directly to the lower-band encoders. It is to be noted 


\section{Tree Encoding in the ITU-T G.711.1 Speech Coder}

that these input signals have been pre-processed by a high-pass filter with a cut-off frequency of $50 \mathrm{~Hz}$.

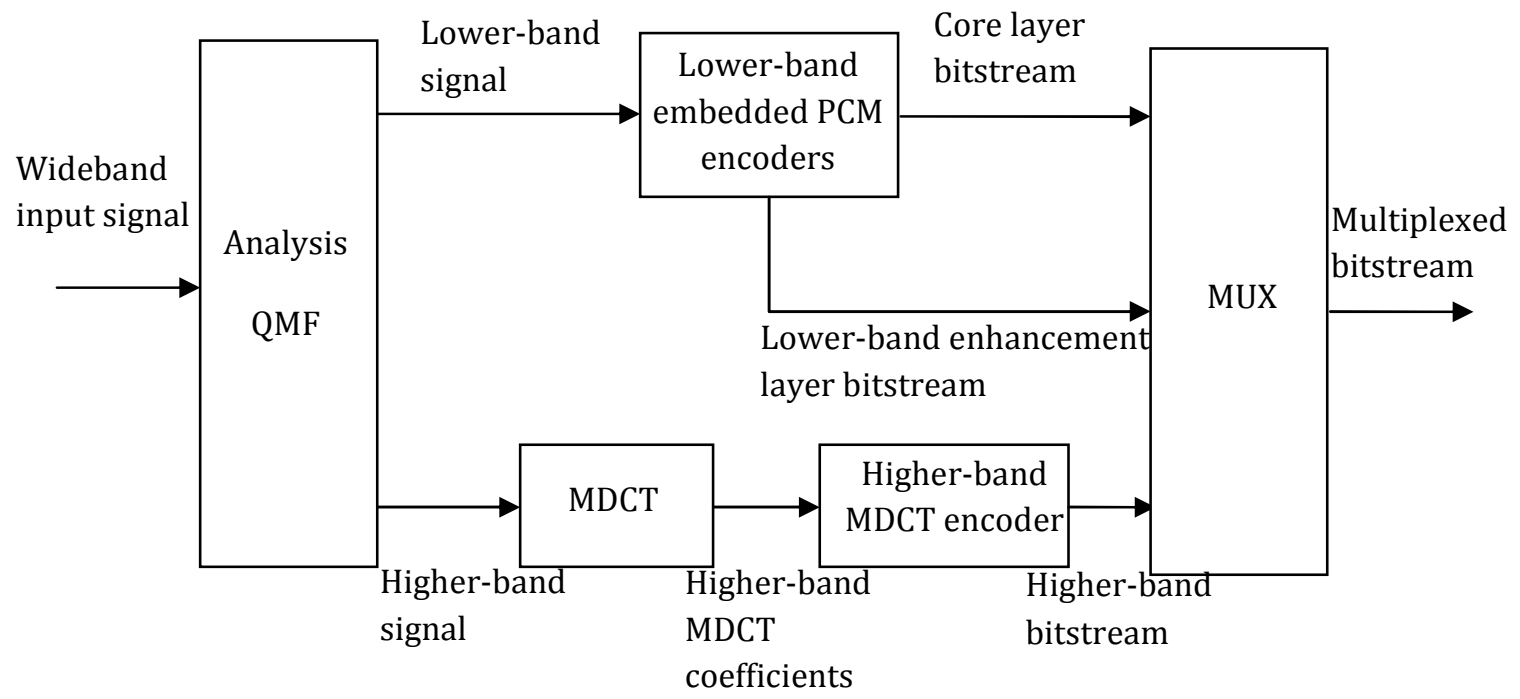

Figure 2-1 - Block diagram of G.711.1 encoder

\subsection{LOWER-BAND ENCODING}

In the lower-band, G.711.1 not only adds noise feedback with perceptual noise shaping to the log companded PCM encoder of G.711, but also an optional enhancement layer to refine the quantization. A local Layer 0 decoder has been added to the design. The locally decoded signal is used for the calculation of the perceptual filter, which then filters the difference between the input signal and the decoded signal. This perceptually shaped noise is then added to the input signal. The resulting signal is quantized by the Layer 0 quantizer and the Layer 0 bitstream is obtained. A refinement signal is sent to the Layer 1 quantizer which generates the 


\section{Coder}

Layer 1 bitstream. The lower-band encoder is show in Figure 2-2. Another addition to the PCM encoder is the concept of dead-zone in which very low energy signals are brought down to the zero level. Essentially it increases the size of the zero quantization region for such signals.

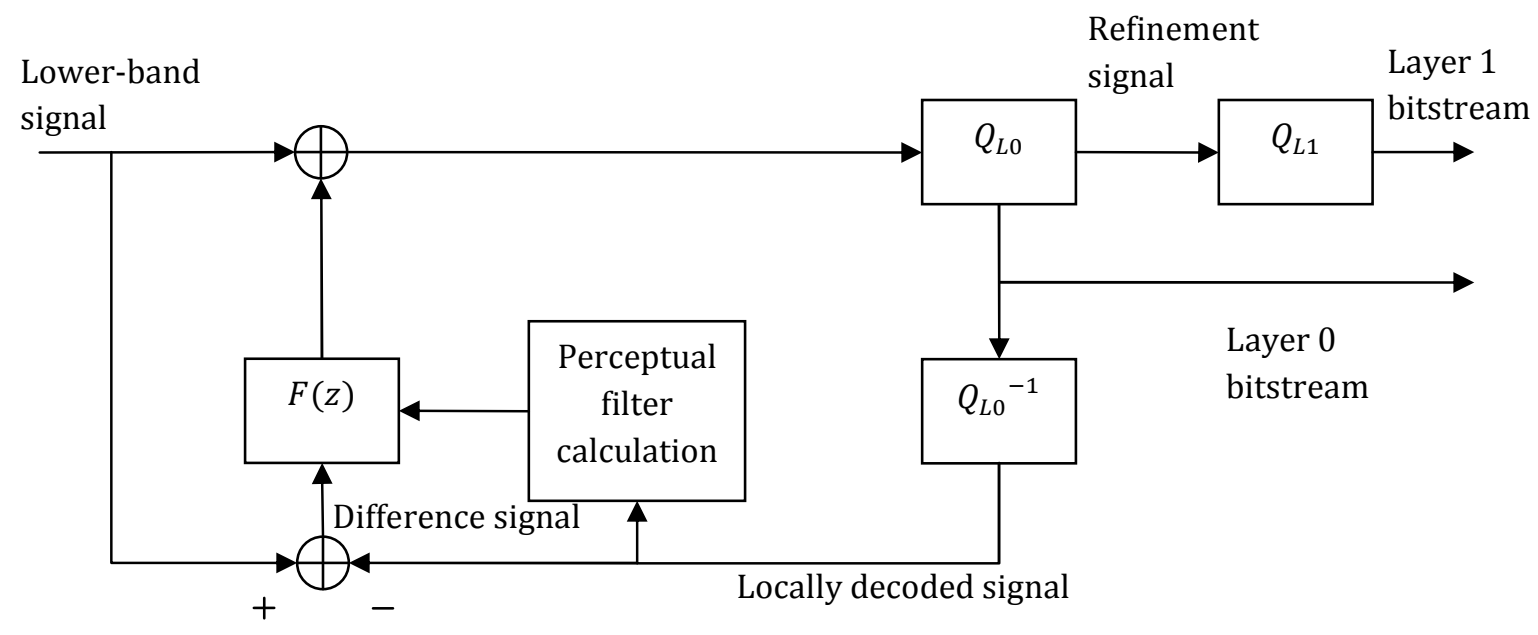

Figure 2-2 - Lower-band encoder

\subsubsection{CORE LAYER}

The core layer can be considered as G.711 with two upgrades. These are, namely, noise feedback and dead-zone quantizer. In the following sub-sections $\mu$-law encoding process, noise feedback and the dead-zone quantizer are further discussed.

\subsection{2 $\mu$-LAW QUANTIZER}

In the $\mu$-law quantizer a 16 -bit sample is coded by a log companded PCM encoder with 8 bits [2]. The bits in the code are allocated as follows:

- One bit for the sign 


\section{Coder}

- Three exponent bits to specify compander segment

- Four mantisa bits to indicate the position within the compander segmet

The coding process takes place sample-by-sample, frame-by-frame. Each frame has 40 samples. The input is 16 -bit, $2 s$ compliment in the range 32,768 to $-32,768$. If $\bar{x}(n)$ is the input sample, the sign given by:

$$
s(n)= \begin{cases}0 \times 80 & \text { if } \bar{x}(n) \geq 0 \\ 0 & \text { if } \bar{x}(n)<0\end{cases}
$$

where $0 x$ represents a hexagonal number. The Layer $0, I_{L 0}(n)$, is 8 -bit index and is calculated as:

$$
\begin{aligned}
& e(n)=\left\lfloor\log _{2}(\bar{x}(n))\right\rfloor-7 \\
& r(n)=\left\lfloor 2^{-e(n) \cdot \bar{x}(n)\rfloor \otimes 0 \times 07}\right. \\
& m(n)=\left\lfloor 2^{-(e(n)+3) \cdot \bar{x}(n)\rfloor-16}\right. \\
& y(n)= \begin{cases}2^{e(n)} \cdot\left(2^{3}(m(n)+16)+4\right)-132 & \text { if } s(n)=0 \times 80 \\
-\left(2^{e(n)} \cdot\left(2^{3}(m(n)+16)+4\right)-132\right. & \text { if } s(n)=0\end{cases} \\
& I_{L 0}(n)=\left(s(n)+2^{4} e(n)+m(n)\right) \oplus 0 \times 7 F
\end{aligned}
$$

where [·] denotes rounding towards minus infinity, $\otimes$ represents AND bit-operator and $\oplus$ represents XOR bit-operator. In the above equations $e$ is the exponent, $r$ is the quantization residual, $m$ is the mantissa, $y$ is the locally decoded signal and $I_{L 0}$ constitutes the Layer 0 bitstream. Instead of transmitting the quantized values, their respective indices in the $\mu$-law coding table are transmitted to the decoder. A copy of 


\section{Coder}

these tables is also available at the decoder and the codes are respectively decoded. It should be noted that $e$ and $r$ form the refinement signal that is sent to the Layer 1 quantizer.

\subsubsection{NOISE FEEDBACK}

The locally decoded signal, $s_{L 0}$, is subtracted from the input signal and the resulting difference is perceptually filtered and added to the new incoming signal. This perceptual filtering makes use of the properties of the human perception system and masks the quantization noise. The perceptual noise shaping filter is based on a linear prediction filter (LP) filter, and is given by [2][4]:

$$
F(z)=A_{0}(z / \gamma)-1
$$

where $A_{0}(z)$ is the fourth order transfer function of the LP filter and $\gamma$ is the perceptual weighting factor.

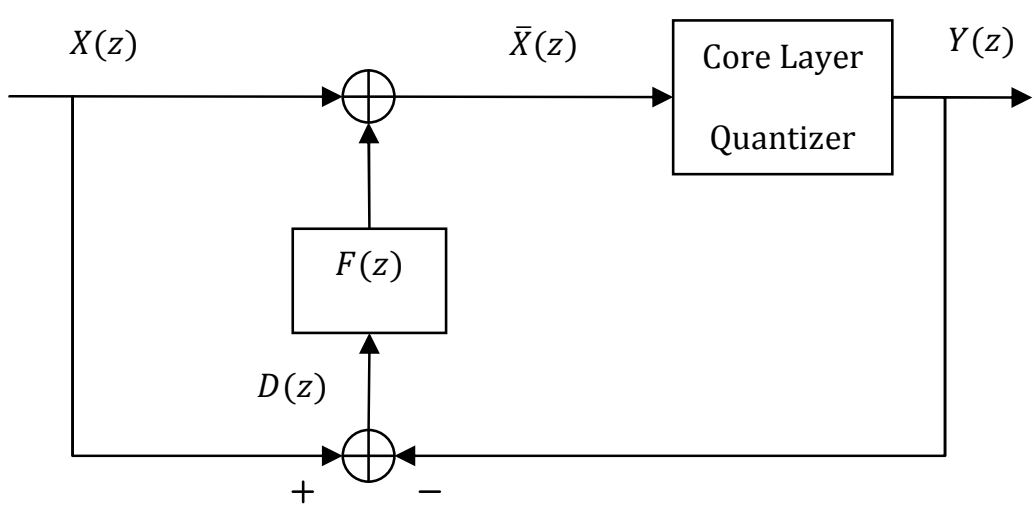

Figure 2-3 - Noise shaping

The filter $F(z)$ needs to be designed such that it perceptually masks the noise. 


\section{Coder}

From Figure 2-3:

$$
Y(z)=\bar{X}(z)+Q(z)
$$

where $Q(z)$ is the quantization noise added at the G.711.1 core layer quantizer, $X(z)$ is the input signal, $\bar{X}(z)$ is the input signal after perceptually shaped noise has been added to it, $Y(z)$ is the locally decoded signal and $D(z)$ is the difference signal.

$$
\bar{X}(z)=X(z)+\{X(z)-Y(z)\} F(z)
$$

From the above two equations, we get:

$$
\begin{gathered}
Y(z)=X(z)\{1+F(z)\}-Y(z) F(z)+Q(z) \\
Y(z)\{1+F(z)\}=X(z)\{1+F(z)\}+Q(z) \\
Y(z)=X(z)+\frac{Q(z)}{1+F(z)}
\end{gathered}
$$

It can be seen that the spectrum of quantization noise is shaped with the spectrum of $1 / A_{0}(z / \gamma)$

A low complexity filter which achieves both formant weighting and controls the tilt in the noise shaping is present in the AMR-WB standard speech codec. Unlike the AMR-WB standard, the filter in G.711.1 speech coder is adaptive. To accomplish the goal of reducing noise between low frequency harmonics, the filter is made dependent on the zero-crossing count [4]. Once the signal has been pre-emphasized, it is windowed to cover both current and previous frames. An asymmetric window is used to strike a balance between simultaneous and pre- and post- masking. The 


\section{Coder}

Levinson-Durbin algorithm is then used to calculate the perceptual shaping filter from the autocorrelation function of the resulting signal. Details of the implementation can be found in [2]. The outcome LP analysis is a filter with the transfer function:

$$
A_{0}(z)=1+a_{1} z^{-1}+a_{2} z^{-2}+a_{3} z^{-3}+a_{4} z^{-4}
$$

After the weighing factor is included, it becomes:

$$
A_{0}(z / \gamma)=1+\sum_{i=1}^{4} \gamma^{i} a_{i} z^{-i}
$$

The noise feedback filter, hence, looks like:

$$
F(z)=\sum_{i=1}^{4} \gamma^{i} a_{i} z^{-i}
$$

Usually a value of 0.92 is chosen for the weighting factor $\gamma$. It is to be noted that this filter is updated after each frame. At the encoder, noise shaping is only applied to Layer 0 . For Layer 1 the noise shaping filter is present at the decoder end. This is to ensure that the shape of the quantization noise is the same when both layers are used as that when only Layer 0 is in operation. As the noise shaping filter is based on the past signals, there is no need to transmit it to the decoder, hence, bandwidth is saved. It can be calculated at the decoder end from the past decoded signal. Details of why the Layer 1 noise shaping filter should be at the decoder end are presented in [4]. They are not listed here as this thesis is primarily concerned with Layer 0. 


\section{Tree Encoding in the ITU-T G.711.1 Speech Coder}

There are two special cases where the noise feedback filter is attenuated. The first case is when very low energy signals are received. The decision to attenuate the filter in such a case based on the normalization factor, $\eta$, calculated as:

$$
\eta=30-\left\lfloor\log _{2}\left(r_{L 0}(0)\right)\right\rfloor
$$

where $r_{L 0}(0)$ is the first autocorrelation coefficient of the pre-emphasized signal from the calculation of the perceptual filter. Because of the limited dynamic range of the G.711.1 quantizer, when a low level signal is received, the perceptual filter will be unable to mask the noise [2]. In this case, when noise cannot be masked, it is best to make it less annoying. A predefined filter is used.

When:

$$
\eta \geq 16
$$

the filter becomes:

$$
F(z)=\sum_{i=1}^{4} 2^{-(i+\eta-16)} a_{i} z^{-i}
$$

This prevents the noise feedback filter from increasing the noise instead of masking it. The second case occurs when signals with energy in higher frequency are received, especially near $4 \mathrm{kHz}$. The noise-shaping feedback might become unstable. This would affect multiple incoming frames before it settles down [2]. Again the filter is attenuated in this case. The first reflection coefficient, $k_{1}$, computed in the Levinson-Durbin algorithm is used to determine this condition. 


\section{Coder}

When:

$$
k_{1}>0.9844
$$

the weighting factor becomes:

$$
\gamma=0.92 \alpha
$$

where $\alpha$ is defined as:

$$
\alpha=16 \cdot\left(1.047-k_{1}\right)
$$

The affect of noise shaping can be seen in Figure 2-4 [4]:
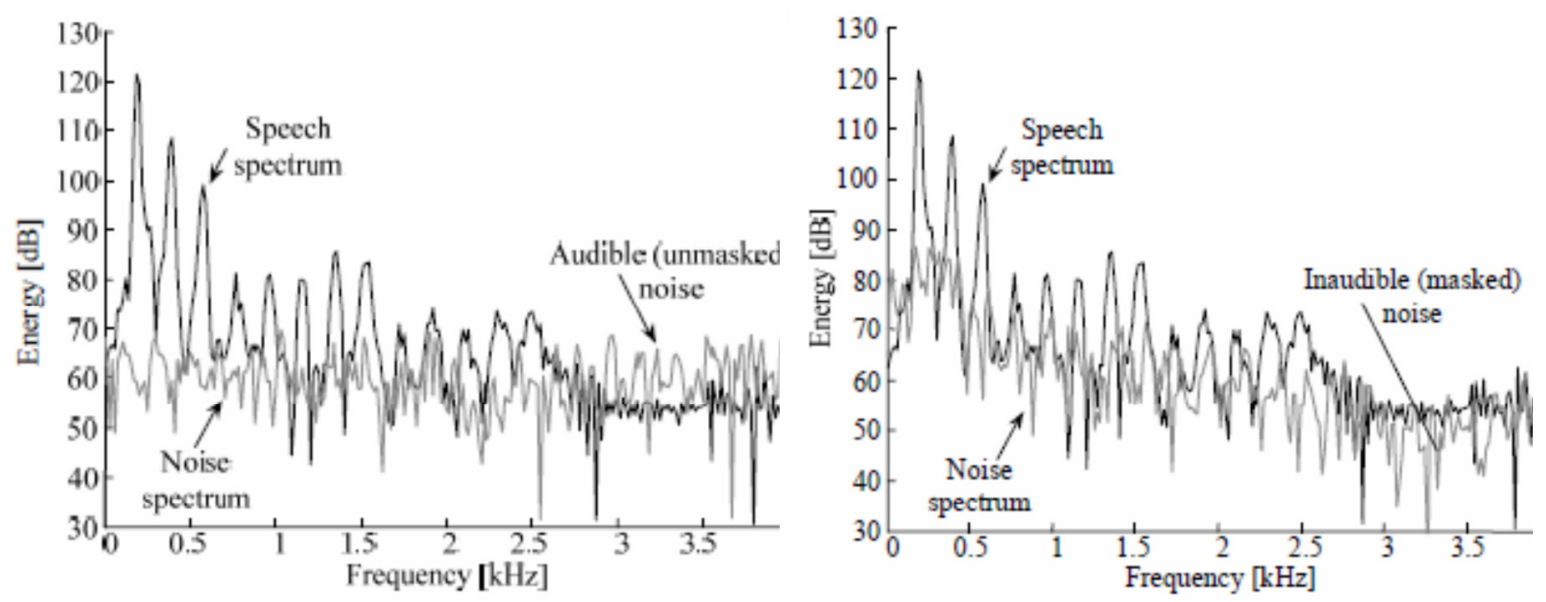

Figure 2-4 - Quantization noise without noise feedback (left) and with noise

\section{feedback (right) [4]}

The noise-feedback filter masks the noise in the speech spectrum, as shown. In the figure on the left hand side it can be seen that the noise on the low frequency end is below the speech spectrum and, hence, inaudible. But in the higher frequency end noise has more energy than the signal and can be heard. With noise shaping, this 


\section{Tree Encoding in the ITU-T G.711.1 Speech Coder}

audible noise in the high frequency range is now masked beneath the speech spectrum. Properties of the human perception system are utilized here. Even though the overall noise energy is higher after filtering, it is inaudible due to masking. Once the difference signal has been filtered, it is added to the new incoming signal.

$$
\bar{x}(n)=x(n)+\sum_{i=1}^{4} \gamma^{i} a_{i} \cdot d(n-i)
$$

The resulting signal is then quantized and the indices transmitted as the Layer 0 bitstream. The difference signal is based on the previous locally decoded signal. It can also be viewed as filter memory.

\subsubsection{DEAD-ZONE QUANTIZER}

The second major addition is the dead-zone quantizer. Like the attenuation in the noise feedback filter, it targets very low energy signals. The lowest quantization steps in a $\mu$-law quantizer are 0 and \pm 7 . Very low level signals, like those of faint ambient noise, can often find themselves high enough to be quantized to the \pm 7 level. This increases the noise in the coded signal. In this case the output of the quantizer is brought down to the zero level. This is done to further perceptually improve the quality of the signal. The dead-zone quantizer is triggered when:

$$
\eta \geq 16
$$

and

$$
-7 \leq \bar{x}(n) \leq+7
$$




\section{Coder}

Once in dead-zone, the output of the quantizer is:

$$
\begin{aligned}
& e(n)=0 \\
& r(n)= \begin{cases}0 & \text { if }-7 \leq \bar{x}(n) \leq-2 \\
2 & \text { if } \bar{x}(n)=-1 \\
4 & \text { if } 0 \leq \bar{x}(n) \leq 1 \\
8 & \text { if } 2 \leq \bar{x}(n) \leq 7\end{cases} \\
& y(n)=0 \\
& I_{L 0}=0 \mathrm{xFF}
\end{aligned}
$$

The resulting quantizer is shown in Figure 2-5 [2]. Decoded value is on the $y$-axis while the $x$-axis represents the input signal. As seen, the dead-zone quantizer "kills" the lowest level and some part of the next level. The dashed line shows the quantizer levels with Layer 1 active. It provides more quantization level options. Though it can quantize with less error, it uses more bandwidth and cannot be used when communicating with a G.711 device. 

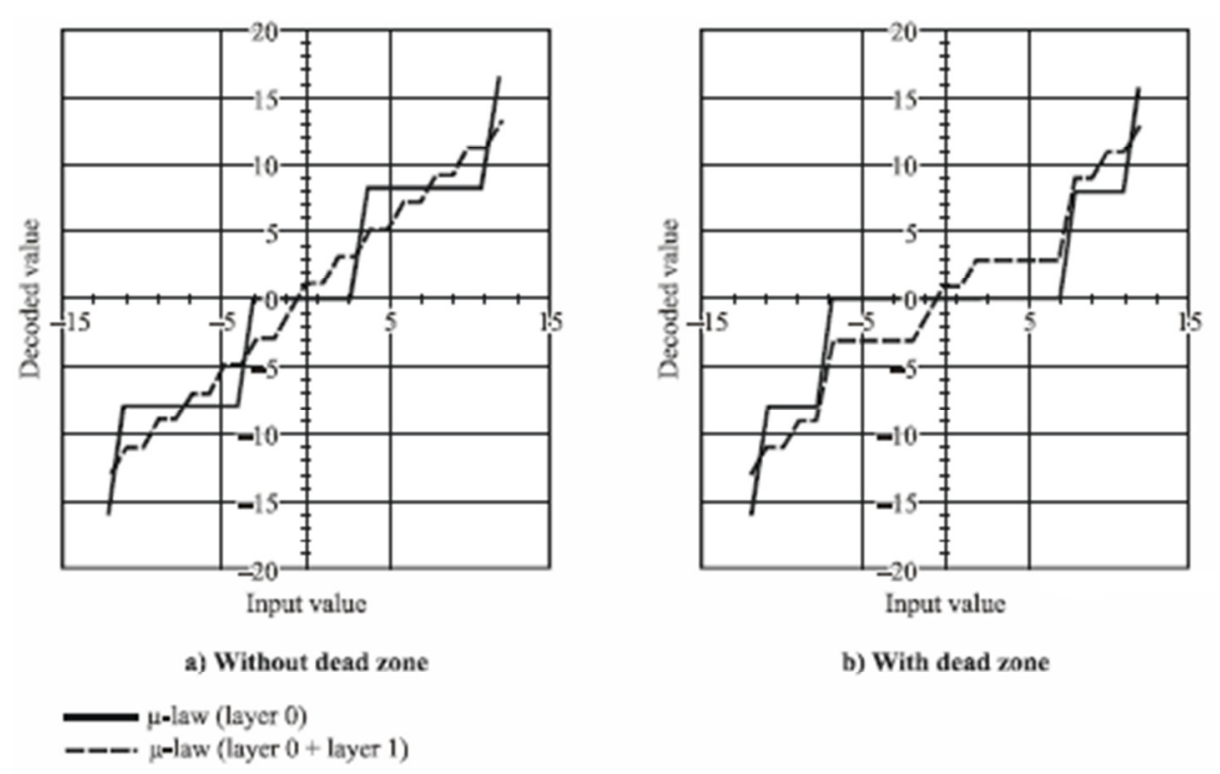

b) With dead zone

Figure 2-5 - Quantization noise without noise feedback (left) and with noise feedback (right) [2] 


\section{Chapter 3 CELP AND VECTOR QUANTIZATION IN ADPCM}

G.711.1, being a log companded PCM coder with modifications, falls in the category of waveform coding. Another similar coder working at a lower bit rate is the DPCM coder. Instead of quantizing the input signal, the DPCM coder takes the difference from a prediction based on the past values and quantizes and codes that. With this the noise ends up being shaped by the synthesis filter. This is solved in ADPCM where feedback is utilized to counteract this noise shaping. In this chapter a basic overview of DPCM and ADPCM coder is provided. Then we go on to discuss CELP coding, a hybrid coder making use of linear prediction and quantizing the residue. Instead of sample-by-sample quantization like the other two coders, CELP employs vector quantization. In the last subsection the structure of the ADPCM is rearranged into a noise feedback version and vector quantization is introduced. It can be seen that such a setting is similar to that of CELP [5]. 


\section{Coder}

\subsection{DPCM}

A DPCM system involves a prediction filter and a quantizer at the coder end and an analysis filter at the decoder end. A high level DPCM block diagram is shown in Figure 3-1.
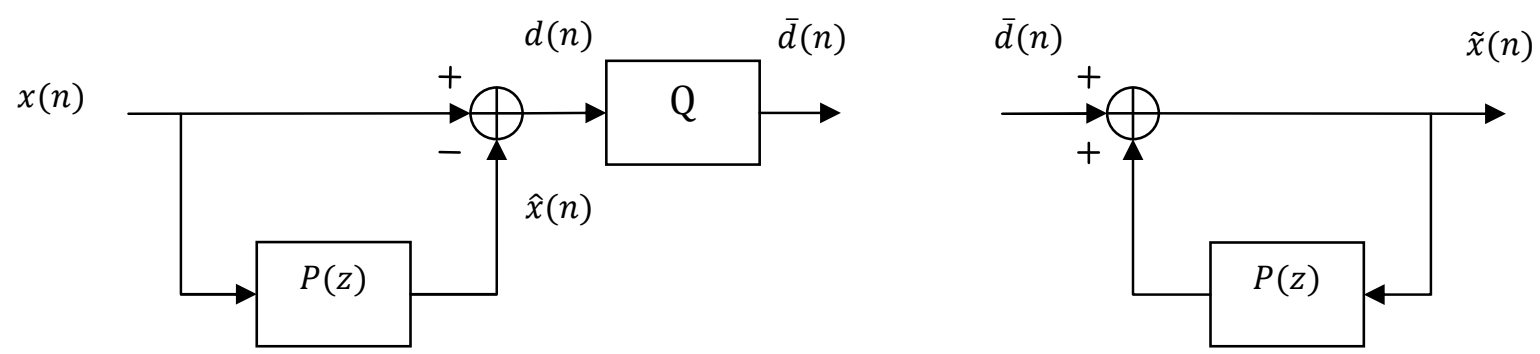

Figure 3-1 - DPCM Coding; encoder on the left, decoder on the right

Based on the past values of the input signal, the prediction filter $P(z)$ creates an approximation of $x(n)$. Usually it is a multi-coefficient filter based on the input signal. It can be computed by solving for the linear predictor coefficients which minimize the mean square error. The difference signal $d(n)$ is then quantized and passed on to the receiver. In an actual scenario indices of the quantization are transmitted and the reconstructed takes place at the decoder end. For simplicity this step is skipped and the quantizer is shown to transmit the reconstructed signal. Analyzing the encoder side it can be seen that:

$$
A(z)=1-P(z)
$$

where $A(z)$ is the analysis filter. The inverse of this, the synthesis filter, is found at the decoder end. Analyzing the decoder: 


\section{Coder}

$$
\begin{gathered}
\tilde{X}(z)=\frac{1}{A(z)} \bar{D}(z) \\
\tilde{X}(z)=\frac{X(z) A(z)-Q(z)}{A(z)} \\
\tilde{X}(z)=X(z)-\frac{Q(z)}{A(z)}
\end{gathered}
$$

where $Q(z)$ is the quantization noise given by:

$$
Q(z)=\bar{D}(z)-D(z)
$$

This shaping of noise by the synthesis filter is undesirable. The solution of this comes in the form of ADPCM.

\subsection{ADPCM}

A feedback structure is employed to adapt to the input signal. The decoder is the same as before, but the encoder is modified, as shown in Figure 3-2.

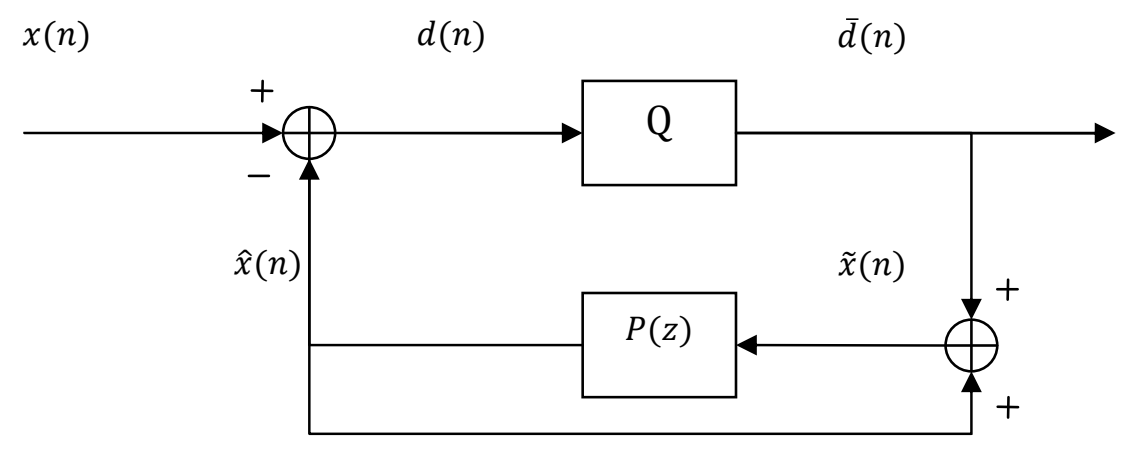

Figure 3-2 - ADPCM encoder block diagram 
The encoder now has a locally decoded signal. Looking at the different relationships between the signals, it can be seen that:

$$
\begin{gathered}
\tilde{X}(z)=\bar{D}(z)+\hat{X}(z) \\
\tilde{X}(z)=D(z)-Q(z)+\hat{X}(z) \\
\tilde{X}(z)=X(z)-\hat{X}(z)-Q(z)+\hat{X}(z) \\
\tilde{X}(z)=X(z)-Q(z)
\end{gathered}
$$

By the addition of the feedback, the noise shaping by the synthesis filter has been removed. The coding process only adds quantization noise, which is white in nature.

\subsection{CELP}

Unlike ADPCM, CELP employs a vector quantizer codebook. As stated earlier, CELP is an analysis-by-synthesis coder. Entries from the codebook are used to synthesize the output at the encoder and compared with the input signal. The entry that gives the best match is selected. The same synthesis filter is used here as in ADPCM. The quantization error is weighted and filtered to give a better perceptual result. A higher level block diagram of a CELP encoder is shown in Figure 3-3. The decoder is again the same.

$W(z)$ is the weighing filter. The codebook keeps a set of possible quantization values for the difference signals for an entire frame. A reconstructed signal from 


\section{Coder}

them is synthesized and compared with the original signal. The quantization error is weighted and perceptually shaped. The mean square error criterion is applied to find the best match. Due to the non-zero internal states, the synthesis and weighting filters have an output even without any input being applied from the codebook. Computations are saved by first calculating this output for the frame and subtracting it from the input signal. After that the response from the codebook input is matched with this new target signal.

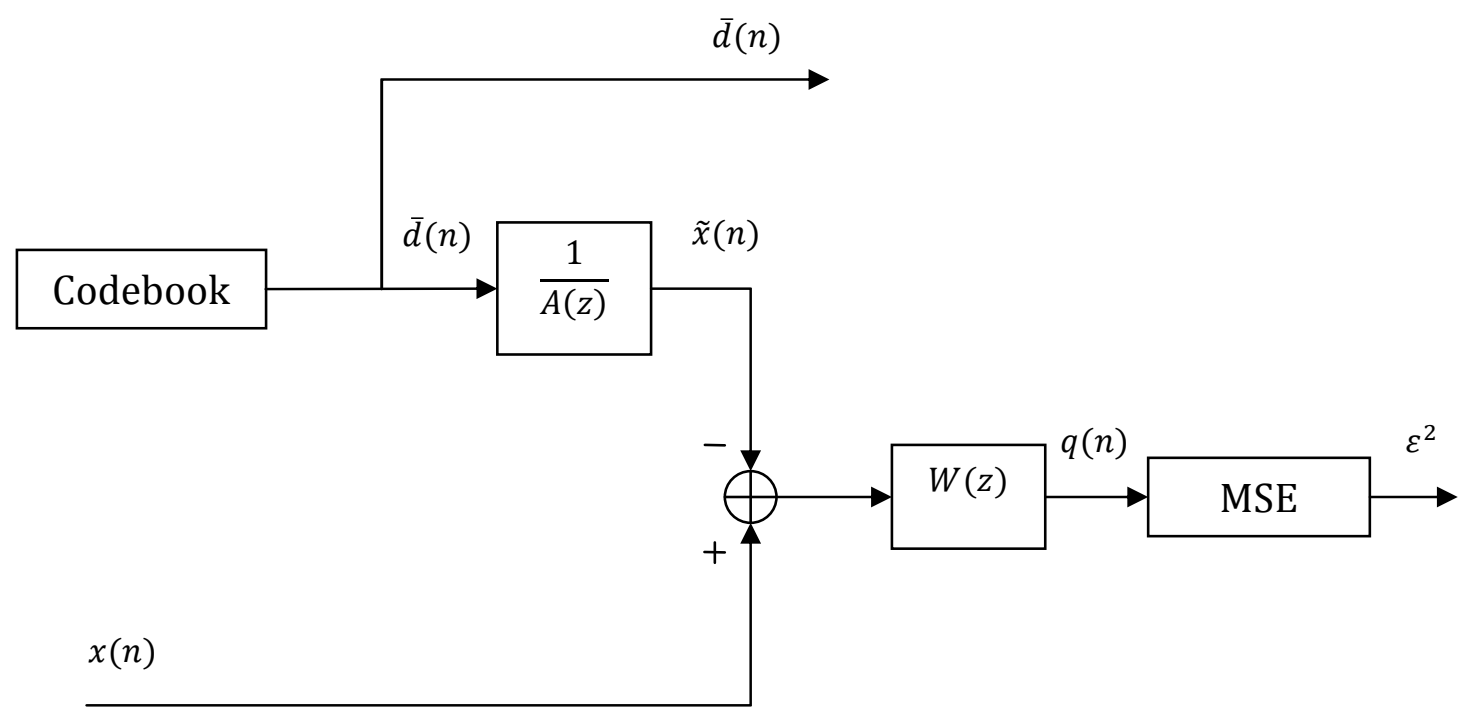

Figure 3-3 - CELP Encoder

$W(z)$ is based on the analysis filter $A(z)$ and shapes the quantization noise. When the analysis filter is based on the LPC filter as described in Chapter 2,1/A(z) can be called the formant synthesis filter. It suppresses the noise between the formant regions of the speech. Generally, the weighting filter can be represented as: 


\section{Coder}

$$
W(z)=\frac{A\left(\gamma_{1} z\right)}{A\left(\gamma_{2} z\right)}
$$

where $\gamma_{1}$ and $\gamma_{2}$ are parameters used to control the shape of the filter.

\subsection{VECTOR QUANTIZATION IN ADPCM}

A CELP coder essentially takes a predicted value, takes the difference from the original input, quantizes the difference, perceptually shapes the quantization noise and makes the decision based on mean square error criterion. It uses the same synthesis filter as ADPCM. ADPCM itself does some noise shaping; it reshaped the quantization noise in DPCM back to white. If the ADPCM structure is further tweaked, the noise shaping property will be further clear. An equivalent structure of the encoder to that of Figure 3-2 is shown in Figure 3-4.

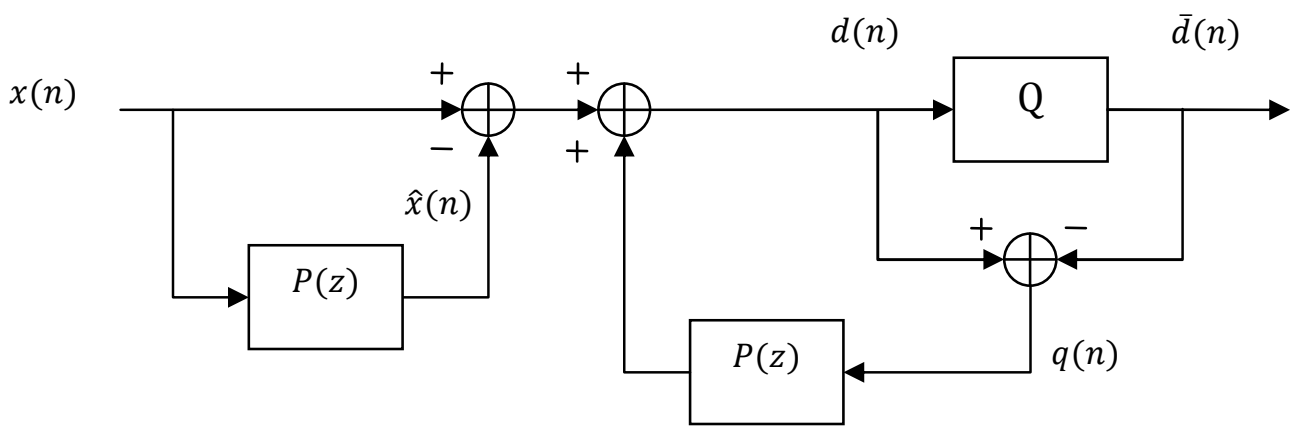

Figure 3-4 - Rearranged ADPCM encoder structure to show noise feedback

The presence of $P(z)$ in the noise feedback path cancels the noise shaping effect of DPCM. If we replace it by a general noise feedback filter, $N(z)$, the noise can be shaped as desired. 


\section{Coder}

$$
\tilde{X}(z)=X(z)-\frac{1-P(z)}{1-N(z)} Q(z)
$$

It would be advantageous if this is made use of and the noise is masked perceptually, a property present in CELP coding. It can be seen that the only major difference left between ADPCM and CELP is the mechanism of quantizing the samples; one is sample-by-sample while the other is vector quantization. Replacing the sample-bysample quantizer in ADPCM by a codebook based VQ, the new structure of ADPCM looks like Figure 3-5.

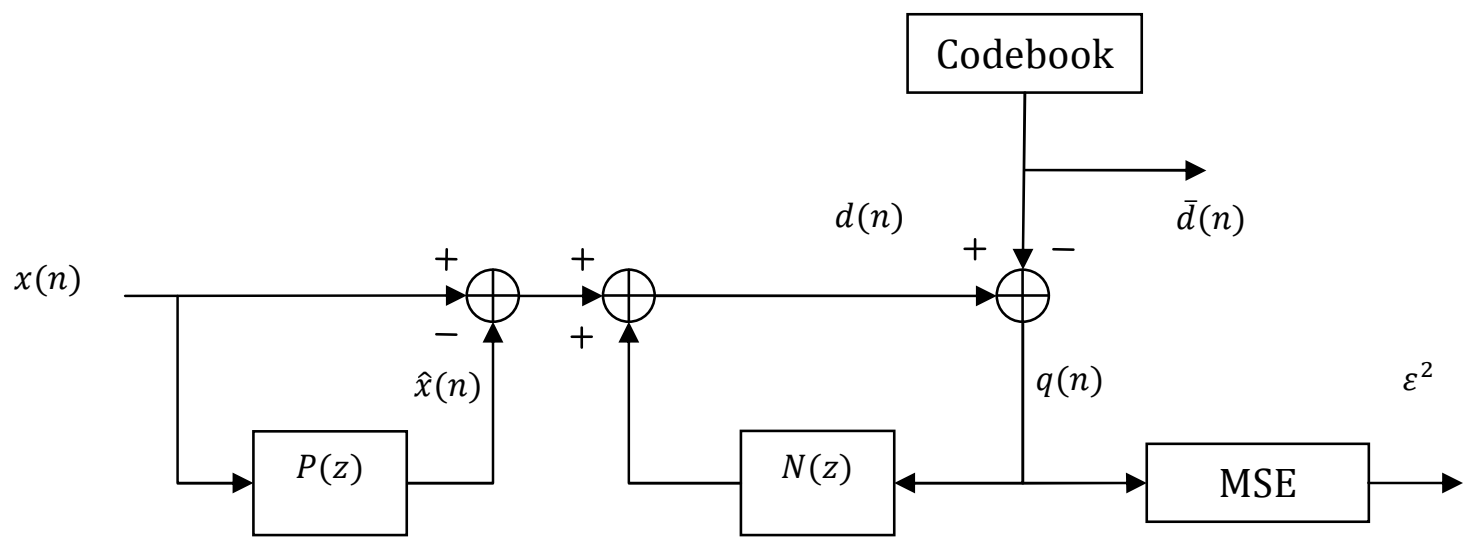

Figure 3-5 - VQ in ADPCM encoder with noise feedback

The encoder can now quantize multiple samples at a time. The codebook consists of all possible quantizer outputs. These outputs are predetermined approximations of the difference signal under the quantization law being implemented. The outputs are compared with $d(n)$. The quantization error, $q(n)$, is fed into the noise feedback loop. The codebook vector with the least error as calculated by the mean square error block (MSE) is chosen and transmitted. Further 


\section{Tree Encoding in the ITU-T G.711.1 Speech Coder}

modifying the structure, we get the arrangements as shown in Figure 3-6 and Figure 3-7.

Form 1 is a rearrangement of structure in Figure 3-5. In form 2 the analysis and noise feedback filters are merged. It can be seen that this is similar to the CELP encoder in Figure 3-3. ADPCM, a waveform coder with a scalar quantization (SQ), has been modified to have noise feedback and vector quantization, just like CELP, a hybrid coder. A similar modification can be performed with the G.711.1 core layer. The benefit is that noise feedback is already present in the new standard; all that needs doing is replacing the quantizer with a similar codebook based vector quantizer which follows the $\mu$-law so that it is compatible with other G.711 devices. It should be noted that these modifications have been done at the encoder side and nothing needs to be done with the decoder as it has remained the same throughout. This goes along with the aim to keep the bitstream G.711 compatible. 


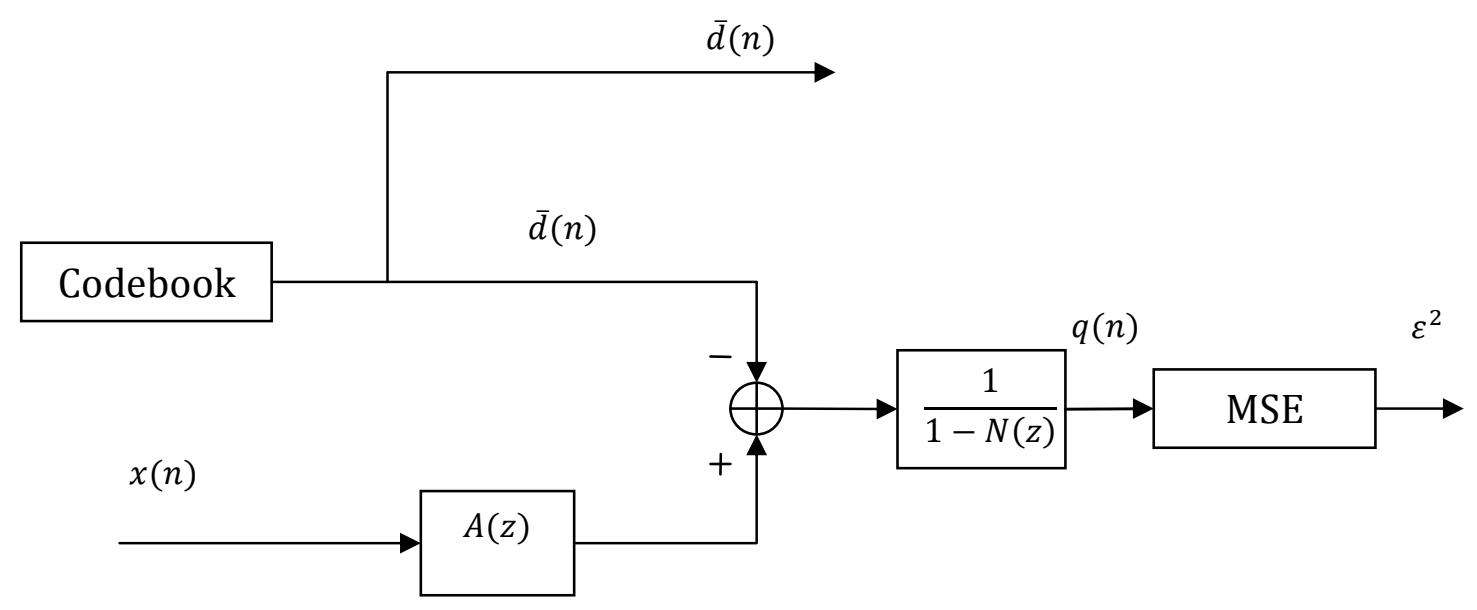

Figure 3-6 - VQ in ADPCM encoder with noise feedback - form 1

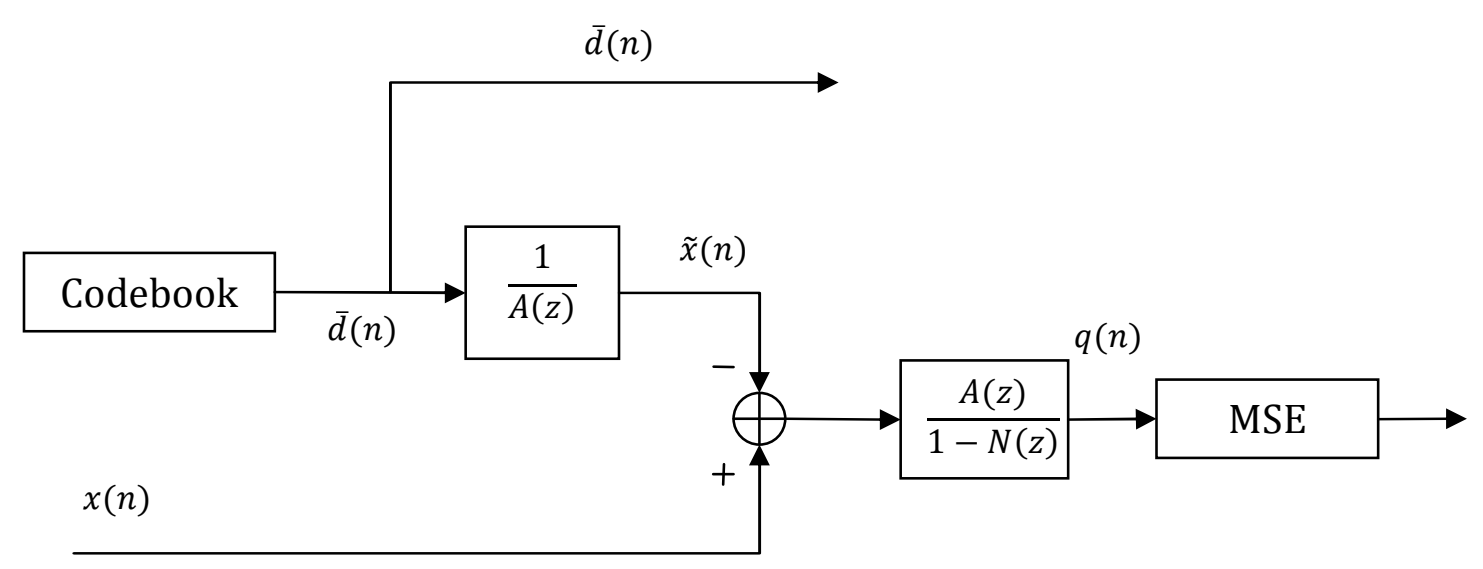

Figure 3-7 - VQ in ADPCM encoder with noise feedback - form 2 


\section{Tree Encoding in the ITU-T G.711.1 Speech Coder}

\section{Chapter 4 DELAYED DECISION CODING}

A vector quantizer takes a batch of input samples and quantizer them at the same time. The aim is the minimization of propagating effect of pervious decision over the whole batch. This approach is better than sample-by-sample quantization as it has a better view of the incoming samples. It is slightly rigid in the sense that it can only make the best possible decision based on the current batch of input samples and is blind to the future inputs and the effects the decision now would have on them. Also when noise feedback is included the effect of pervious decision can propagate further, even increase, due to filter memory. As mentioned earlier the CELP filters already have a zero input response. This is beyond the control of the quantizer as its scope is limited to the current set of input samples. In a CELP coder an entire $5 \mathrm{~ms}$ frame ( 40 samples) is processed at the same time by the vector quantizer. Due to the large set of samples the effect of this propagating error is not that profound. A $\mu$-law quantizer already has 256 quantization levels. To replace it by a vector quantizer, multiple samples have to be quantized at the same time. The vector quantizer codebook tremendously increases in size even when one more sample is added $(65,536$ codebook entries for two samples). To keep the complexity 


\section{Tree Encoding in the ITU-T G.711.1 Speech Coder}

low, only two samples are quantized at the same time. Hence, the propagation of error due to noise feedback and filter memory will have a much greater effect. To counter that delayed decision coding is suggested. A coding technique which waits for further samples to arrive, evaluate the effect of different decisions on these future samples and then makes the best possible decision. If a vector quantizer can be viewed as jumping from frame to frame, delayed decision coding can be viewed as sliding across the frames.

\subsection{TREE ENCODING}

One such delayed decision coding method is tree encoding. A tree is populated with different possible decisions when new samples are received. Cumulative errors over the branches are taken into consideration. Once a decision has been made, the tree is pruned to keep the complexity under control and to remove the branches which will not be further expanded. Examples of tree encoding can be found in [3], [6] and [7].

\subsubsection{SINGLE PATH TREE ENCODING}

Single path tree encoding is much simpler than multi-path tree encoding. It is being mentioned over here to describe some tree encoding terms which are common in both. Three important terms are associated with tree encoding:

- Nodes

- Branches

- Leaves 


\section{Tree Encoding in the ITU-T G.711.1 Speech Coder}

A node is a time instant which has a quantizer output associated to it. For a single path tree encoder a tree is only left with one node once a decision has been made. The quantizer output associated with it is the best possible approximation of the input samples based on the error criterion. Whenever new samples are received and decision has to be made, the tree is expanded from this node. For case of a two sample $\mu$-law vector quantizer, 65,536 branches stem from it. At the end of each branch is a leaf. The leaf holds the possible quantizer values which could be selected for this time instance. Once the best possible match has been selected, the selected leaf becomes the node for the next round and the rest of the leaves are discarded. Therefore, only one path is kept. The tree is continuously populated and pruned, and in the end one single path is left which defines the code. There is no delay in the coding of the samples. The code can be transmitted as soon as the decision is made. This type of coding can be seen in CELP. If a vector quantizer is replaced by a scalar version, it can also be seen in PCM encoders. 


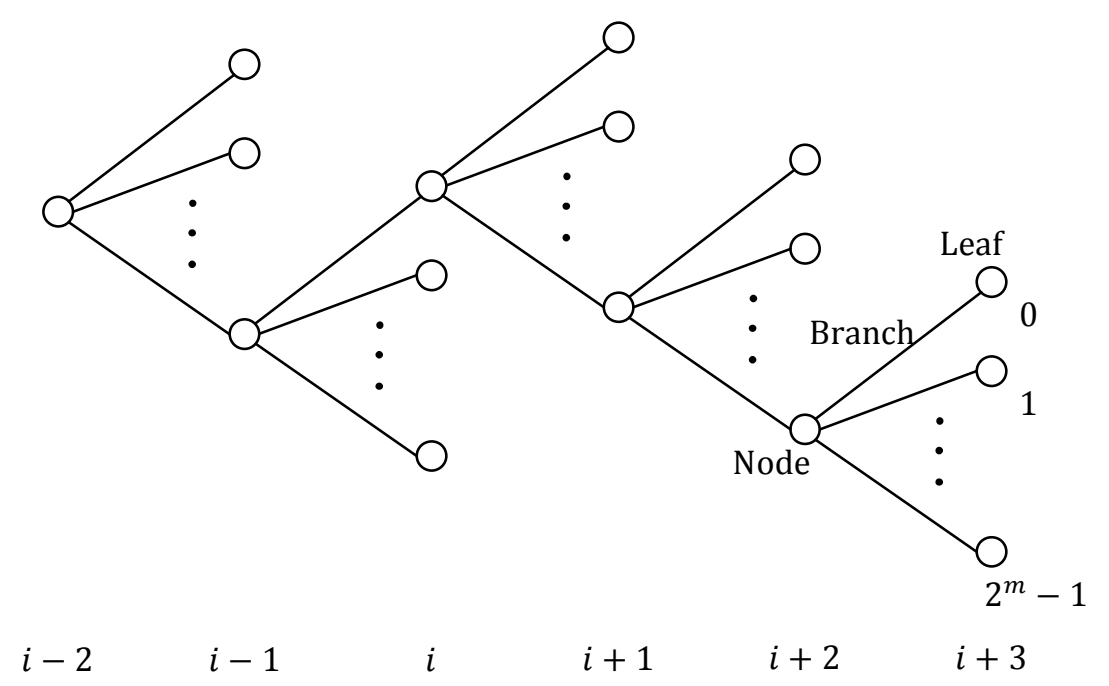

Figure 4-1 - Single path tree encoding

\subsubsection{Multi-PATH TREE ENCODING: THE $(M, L)-A L G O R I T H M$}

In a single path tree encoder only one node is available each time the tree is branched out. There is no delay in making the decision as the code can be transmitted almost instantaneously. If an artificial delay is added and the decision is reserved till its effect on further decisions can be evaluated, multi-path tree encoding is realized. The tree is branched from multiple nodes and, therefore, many more leaves are available to choose from. The $(M, L)$-Algorithm is used to implement the multi-path tree encoder. This algorithm is similar to the one implemented in [3]. 


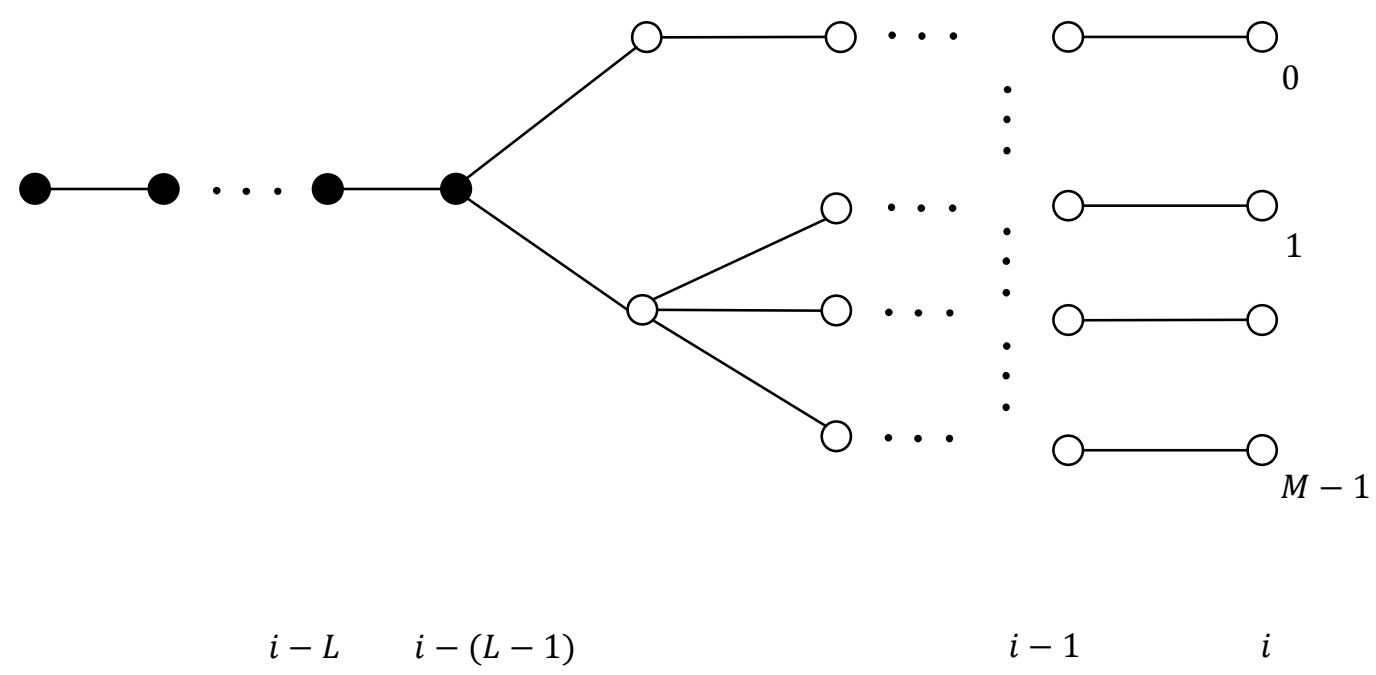

Figure 4-2 - Multi-path tree encoding

This algorithm is defined by the two parameters $M$ and $L . M$ is the spread of the tree. Essentially it is the maximum number of nodes to be kept behind after a decision has been made and the tree pruned. $L$ is the depth of the tree. It is the number of branches in series which define the possible selection paths. A trellis has a constant number of nodes after the initial exponential expansion. On the other hand the tree, under the $(M, L)$-Algorithm, grows gradually and is constantly pruned to keep its growth under check. It can also be classified a search algorithm which finds the best suitable path, based on the error criterion, under the two constraints of maximum number of nodes, $M$, and tree depth, $L$. A tree under the $(M, L)$-Algorithm is shown in Figure 4-2. 


\section{Tree Encoding in the ITU-T G.711.1 Speech Coder}

After the $i^{t h}$ input block has been processed, a maximum of $M$ nodes are kept. There is an equal number of paths present as each node signifies one path. If traced backwards it can be seen that all these paths converge back to a node at time $i-(L-1)$. Hence, when the $i^{t h}$ input block has been processed, the decision has been made on the $(i-(L-1))^{t h}$ node. The code for that block is transmitted. Therefore, an artificial delay of $L-1$ is created.

At the next instance when $(i+1)^{s t}$ block is input, each of the $M$ nodes is populated with $2^{m}$ number of nodes. For a $\mu$-law vector quantizer working on two samples the code book has 65,536 entires. Hence, $m$ is 16 . At the end of each branch is a leaf, which has a possible quantizer value associated with it. As compared to the single path tree encoder, $M$ times more output choices are available. The nodes are populated with the same set of codebook entries, but because each branch originates from a different node, which has a different quantizer value associated with it, all the new leaves are different and unique. Each path has its own error associated with it, and the filter states on each path are different as well. To ensure this uniqueness it has to be made sure that when the tree is pruned after a decision making instance, each of the $M$ paths that are left behind is different. Some tree encoding implementations might require that the branch numbers be transmitted [3], but in this case the bitstream needs to be G.711.1 compliant. Hence, the indexes of the quantizer decisions are sent. Therefore, the fact that there are different branches which have the same branch number because all the nodes have been expanded from the same codebook does not interfere with the coding process. 


\section{Tree Encoding in the ITU-T G.711.1 Speech Coder}

Once the nodes have been populated, the leaf with the best quantization output associated with it according to the cumulative error criterion, to be described later, is chosen. Once this selection, at time $i+1$, is done, the branch is traced back to the time $i-(L-2)$ and the node which leads to this selected leaf at time $i+1$ is chosen as the best code for the $(i-(L-2))^{t h}$ input block. The codebook index for the quantization value associated with this node is, hence, transmitted. After this, the tree is pruned and a maximum of $M$ paths are selected and kept behind. The path linking the leaf which was selected to have the best quantization output associated with it at the time $i+1$ and the optimal node for the time instance $i-(L-2)$ is always included. It has to be ensured that all of the paths have to converge to the newly selected optimal node for the time instance $i-(L-2)$. This is to maintain the continuity of the optimal path. The $M$ paths which are kept behind are based on the cumulative error. This encoding process continues as further blocks are input.

There is an upper bound on the number of branches that can be kept behind. The maximum number of nodes in a tree, for a depth of $L$ are $2^{m(L-1)}$. Therefore,

$$
M \leq 2^{m(L-1)}
$$

There are two special cases of multi-path tree encoding. The first one is when $L=1$. In this case $M=1$ as well and single path tree encoding is realized. When $M$ is at its upper bound, all possible paths are considered. Even though this is the optimal approach, it increases the complexity drastically. Hence, the value of $M$ is kept less than $2^{m(L-1)}$. Even though this is not optimal, enough paths are considered to 


\section{Tree Encoding in the ITU-T G.711.1 Speech Coder}

provide a near optimal solution while keeping the complexity low. The other special case is when $M=1$. In this case only one node is kept back after the decision has been made. There is no point in keeping $L$ larger than 1 because there is only one single path. Increasing the tree depth would only add delays without any benefits. Hence, when either $M$ or $L$ is 1 , the other is as well.

\subsection{CUMULATIVE ERROR}

The error measure decides how the tree is populated and in turn pruned. Hence, it plays a vital role in tree encoding. The benefit of a tree encoder is that it looks at future values and sees how a decision made now will have an effect on them. To make use of this property it is only wise to use an error measure which looks at long term distortions. Therefore, the cumulative error over the whole path is chosen to be the error measure. To be more specific, the cumulative sum of the mean square error of all nodes in the path is considered. At the time instant $i+1$ decision is made for the code for the input block at time instant $i-(L-2)$. It is chosen such that:

$$
E_{\text {opt }}=\min _{j}\left[\sum_{k=0}^{i+1} e_{j}^{2}(k)\right] \text { for } 0 \leq j \leq t-1
$$

where $E_{\text {opt }}$ is the cumulative error of the chosen path, $e_{j}^{2}(k)$ is the mean square error at a node at the $j^{\text {th }}$ branch at time instance $k$ and $t$ is the number of paths available at time $i+1$. As all the paths originate from the already chosen node at time $i-(L-1)$, the cumulative error till that point is common to all paths. This can eliminated and the equation for the optimal cumulative error is modified to: 


\section{Coder}

$$
E_{\text {optimal }}=\min _{j}\left[\sum_{k=i-(L-2)}^{i+1} e_{j}^{2}(k)\right] \text { for } 0 \leq j \leq t-1
$$

\subsection{MODIFICATION TO G.711.1 CORE LAYER}

In Chapter 3 it was shown how ADPCM coding can be similar to CELP coding with the inclusion of vector quantization and generalizing the noise feedback filter. A similar case can be developed for G.711.1 core layer. As the G.711.1 core layer is based on PCM coding instead of ADPCM, the analysis and synthesis filters are excluded. Noise feedback coding has already been incorporated into the new standard. By replacing the quantizer with a codebook based VQ, G.711.1 core layer looks like Figure 4-3.

The codebook is fed with the error from the MSE block to help in making the correct decision. This structure can be rearranged to have it look more like the CELP structure shown earlier. Figure 4-4 depicts this rearrangement. 


\section{Coder}

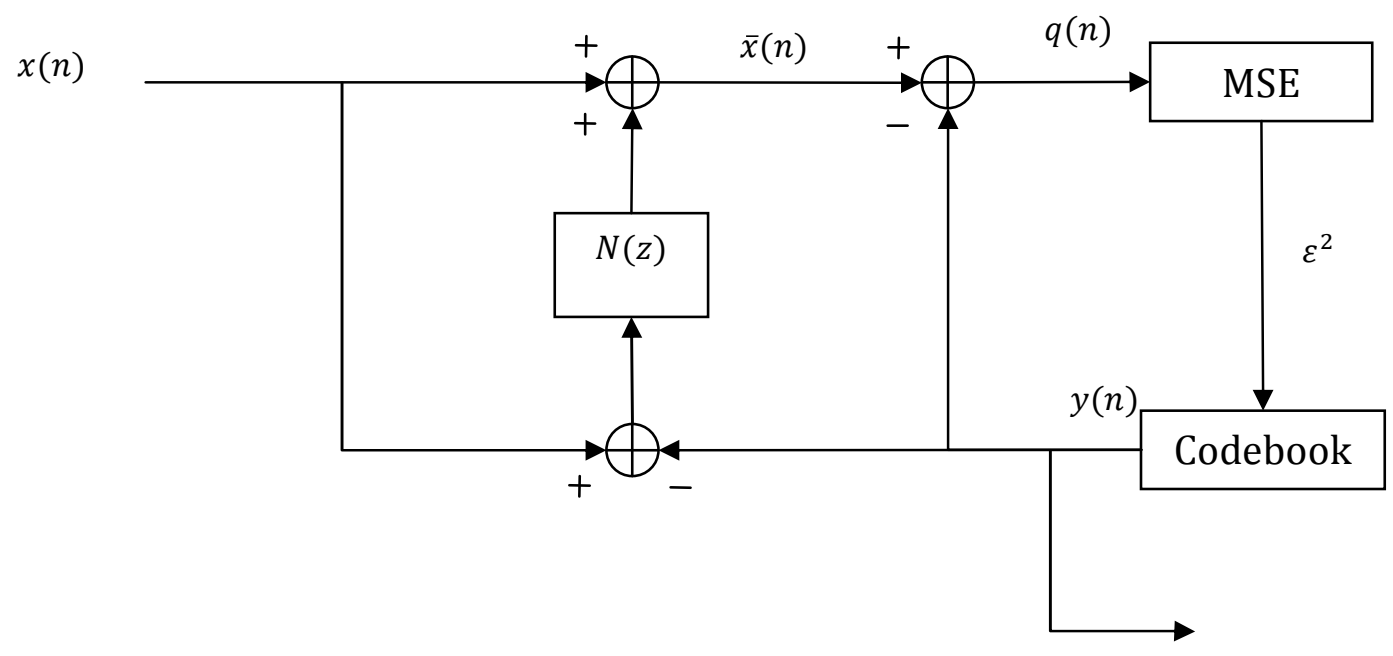

Figure 4-3 - G.711.1 core layer with codebook VQ

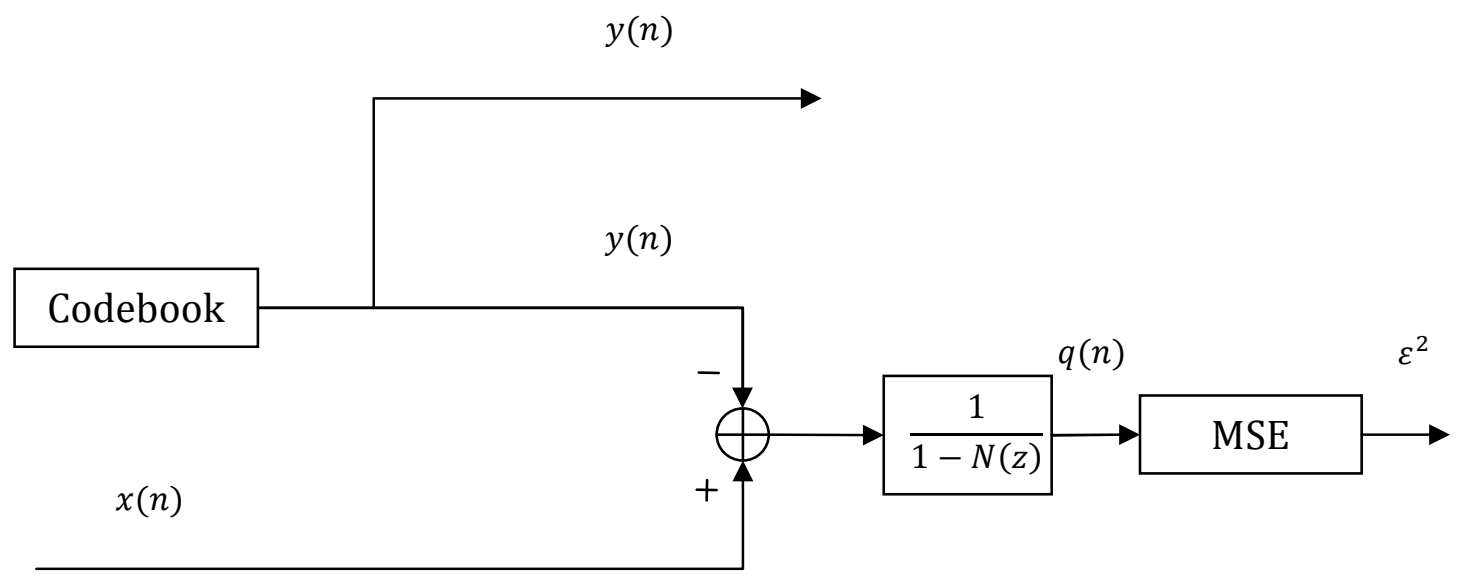

Figure 4-4 - G.711.1 core layer with codebook VQ - rearranged

Again it is seen that it has a similar structure, only the analysis and synthesis filters are missing as G.711.1 works on the original input signal without making any prediction. G.711.1 already has the weighting filter built into it as the noise feedback 


\section{Tree Encoding in the ITU-T G.711.1 Speech Coder}

filter. It is based on the human perception system and shapes the noise accordingly. Therefore, there is no need to modify that. Tree encoding was chosen because a vector quantizer does not care about the effect its decisions have on the future input values due to the filter memories. In a $\mu$-law codebook vector quantizer only a few samples can be quantized at the same time due to complexity concerns as an increase of one more in the block size increases the codebook size 256 times its previous size. Therefore, the block size has to be kept small. With a smaller block size there are more decision instances, hence, there are more instances when the quantizer is ignorant of the effect its decision would have on the incoming samples. To overcome this short coming, delayed decision coding, tree encoding to be more precise, has been introduced. Once implemented the G.711. core layer looks like the tree in Figure 4-2 with each new leaf having a modified G.711.1 core layer encoder like that of Figure 4-3 (or Figure 4-4 as they are both the same) on it, with the difference that each leaf only has one codebook entry associated with it and the error is not fed to the codebook. The $(M, L)$-Algorithm is then employed. 


\section{Chapter 5 Computer Simulation}

Until now the theories behind the system have been discussed, and the structure of the modification to be performed. In this chapter the computer simulation of the encoder will be explained. The simulation was performed on a Dell Studio Desktop, a Quad-core Core 2 Quad 2.8 GHz, 8GB RAM computer running Windows Vista 64-bit edition. The programming has been done in MatLab. In the initial sub-sections the sub optimization of the codebook to reduce the complexity of the encoder, the initialization of the system and the simulation inputs are discussed. Later on a performance evaluation method, perceptual evaluation of speech quality (PESQ) [9], [10], is described and the simulation results provided. The performance of both vector quantized G.711.1 core layer and tree encoded G.711.1 core layer is compared with that of the G.711.1 core layer as in the ITU-T standard. Later on performance of the tree encoder as $M$ and $L$ are varied is provided for further insight.

\subsection{SUB OPTIMAL APPROACH TO REDUCE COMPLEXITY}

Complexity is a very important parameter of a speech encoder. It is directly related to the size of the codebook. A $\mu$-law encoder has 256 levels, for each input 


\section{Tree Encoding in the ITU-T G.711.1 Speech Coder}

sample. Hence, for each additional sample in the input block, the codebook size increases 256 times. To keep the codebook from having an enormous size the size of the input block has been restricted to 2 . This means the codebook has 65,536 entries. This is still a very large size as compared to a typical CELP codebook (1024 entries). To cut down on it, a sub optimal approach is proposed. For each input block instead of looking at the entire codebook to find the optimal match, the search is performed in the local neighbourhood of the input samples. For this purpose the input block is first quantized by a scalar $\mu$-law quantizer, without the addition of noise feedback. This is done by using tables to cut down on the processing time. Once quantized, the neighbouring quantization intervals are chosen as the sub optimized codebook for the population of the tree. The neighbourhood need not be large as a $\mu$-law quantizer has pretty large quantization intervals. The neighbour hood is chosen to be \pm 2 samples of each input sample. That makes 5 choices for each input sample, including itself. With a block size of 2 the sub optimized codebook has a size of 25 .

In the G.711.1 core layer there are two major operations. There is one quantization operation and one filtering operation. With a vector quantizer there is one quantization operation but the number of filtering operations is increased to the size of the sub optimized codebook, which is 25 , as each entry has to be filtered. In a tree encoder there is still only one quantization operation but the number of filtering operations is now $M$-times the size of the sub optimized codebook, because all the $M$ paths that have been kept behind have to be branched. It should also be 


\section{Tree Encoding in the ITU-T G.711.1 Speech Coder}

noted that even though the complexity of each filtering operation in a vector quantizer and tree encoder is twice that of G.711.1 core layer, because 2 samples are being coded, the per sample complexity of each filtering operation is still the same. The filtering operation is the main resource consuming activity. In G.711.1 each filtering operation, per sample, has 4 multiplication operations and 3 addition operations. The vector quantizer has 25 times that many. For a tree encoder that figure is further increased by $M$-times. Also in vector quantization and tree encoding after each filtering operation mean square error is calculated. Each mean square error calculation for two samples requires 2 multiplication operations and 3 addition operations.

For a typical value of $M=3$, the increase in complexity for tree encoding is substantial. G.711.1 has considerable processing power which it requires for the lower-band enhancement layer and the higher-band layer. When working at $64 \mathrm{kbps}$ only the core layer is present. All the processing power available for the other two layers does not get utilized. As tree encoding only works with the core layer, in this certain scenario it can be turned on to make use of the already present processing power, which would otherwise remain unused. 


\section{Tree Encoding in the ITU-T G.711.1 Speech 2010 Coder}

\begin{tabular}{|l|c|c|}
\hline Encoder & Multiplication operations & Addition operations \\
\hline G.711.1 Core Layer, 64 kbps & 4 & 3 \\
G.711.1 Core Layer with VQ, & $25 \times 4+25 \times 1$ & $25 \times 3+25 \times 3 / 2$ \\
$64 \mathrm{kbps}$ & & \\
$\begin{array}{l}\text { G.711.1 Core Layer with Tree } \\
\text { Encoding, 64 kbps }\end{array}$ & $M \times(25 \times 4+25 \times 1)$ & $M \times\left(25 \times 3+25 \times \frac{3}{2}\right)$ \\
\hline
\end{tabular}

Table 5-1 - Multiplication and addition operations per sample of different

\section{G.711.1 encoders}




\section{Tree Encoding in the ITU-T G.711.1 Speech Coder}

\subsection{INITIALIZATION OF THE SYSTEM}

The initial state of the system is very important and affects the performance. A good many variables define this initial state. These include filter memories, feedback filter coefficients and initial multi-path tree.

The filter memories and feedback filter coefficients are all set to zero for ease. In the simulated implementation the multi-path tree is represented by a three dimensional tree. The rows represent different nodes at a certain time instance while the columns hold different variables associated with that node. The third dimension represents the different time instances. The variables associated with each node are:

- Quantized value of first sample

- Quantized value of second sample

- Cumulative error till that node

- Row number of previous node in the path

- Noise feedback filter memory

Each node needs to know its predecessor so that the optimal path can be traced back. Each decision has its own effect on the future values by altering the noise feedback filter memory. Therefore, it is vital to keep track of it. All of these variables are initialized to zero. This means at the initialization the tree is essentially a single path and branches out to $M$ nodes for the last decision making instance. 


\section{Tree Encoding in the ITU-T G.711.1 Speech Coder}

\subsection{SIMULATION INPUTS}

As input to the system four speech sentences were used. They were recited by two different speakers, one male and one female. The sentences are:

- The empty flask stood on the tin tray.

- A speedy man can beat this track mark.

- It is easy to tell the depth of a well.

- These days a chicken leg is a rare dish.

Each speaker recited two consecutive sentences. The male speaker recited the first two sentences while the female speaker recited the last two. All of these samples are combined into one input signal, sampled at $8 \mathrm{kHz}$.

\subsection{PERFORMANCE}

Performance results are listed in this sub-section. The performance has been evaluated using the performance evaluation method PESQ. A brief explanation of this is method is provided in the following sub-section.

\subsubsection{PERCEPTUAL EVALUATION OF SPEECH QUALITY}

The standard ITU-T P.862 [10] is an objective method to assess the end-toend speech quality of a narrow band speech coder. To evaluate the coder, the original input signal to the encoder and the output from the decoder is compared. The result is a prediction of the perceived quality. The amplitude of both the signals is adjusted and brought to a standard level. The degraded signal (output from the 


\section{Tree Encoding in the ITU-T G.711.1 Speech Coder}

decoder) is then time aligned with the original signal. Delays during both silence periods and speech periods can be handled by the algorithm. After that both the original signal and the time aligned degraded signal are transformed into an internal representation. The transformation is such that to match the auditory system of the humans. The transformation has different steps which include Bark spectrum calculation, frequency equalization, equalization of gain variation and loudness mapping. Once both the signals have been transformed by this perceptual model, the difference is passed through a cognitive model. The output is similar to that of MOS scores. The output range of the score is -0.5 to 4.5 . The output score is based on the two parameters that are calculated by the cognitive model [9], [10].

$$
\text { PESQ Score }=4.5-d_{s}-0.0309 d_{a s}
$$

where $d_{s}$ is the symmetric disturbance and $d_{a s}$ is the asymmetric disturbance as calculated by the cognitive model.

\subsubsection{COMPARISON WITH G.711.1}

According to the theory, both the quantizer modified with codebook based VQ and delayed decision coding implemented by the $(M, L)$-algorithm should perform better than the original G.711.1 core layer quantizer. To evaluate this comparison has been made at two different signal power levels. In the first scenario the signal is fed in without any attenuation. The quantizers are not saturated and use as many of the quantization levels available as possible. In the second scenario the power of the signal is attenuated by $-40 \mathrm{~dB}$ to force the quantizer to use fewer 


\section{Tree Encoding in the ITU-T G.711.1 Speech Coder}

quantization levels. This is done to evaluate the performance of the encoders at lower $S N R$ and increased quantization noise. The PESQ scores for the three cases under the two scenarios are listed in table 5-2. Scores for G.711.1 core layer with lower-band enhancement layer switched on are provided as well.

\begin{tabular}{|l|c|c|}
\hline Encoder & Signal without attenuation & Signal at $-40 \mathrm{~dB}$ attenuation \\
\hline $\begin{array}{l}\text { G.711.1 Core Layer, 64 kbps } \\
\text { G.711.1 Core Layer with VQ, }\end{array}$ & 2.263 \\
64 kbps & 4.252 & 2.616 \\
$\begin{array}{l}\text { G.711.1 Core Layer with Tree } \\
\text { Encoding }(M=3, L=3), 64 \\
\text { kbps }\end{array}$ & 4.314 & 2.625 \\
$\begin{array}{l}\text { G.711.1 Core Layer with } \\
\text { Lower-band Enhancement }\end{array}$ & & \\
Layer, 80 kbps & 4.421 & 3.310 \\
\hline
\end{tabular}

\section{Table 5-2 - Comparison of different G.711.1 encoders using PESQ}

At $64 \mathrm{kbps}$, as expected, the tree encoder provides the best result, with the vector quantizer being better than the G.711.1 core layer. This holds true for both the signals, with and without attenuation. With the luxury of coding more than one sample at the same time, both the VQ and the tree encoder are able to make better decisions. The tree encoder is further able to improve by looking at future values.

With the availability of more data rate ( $80 \mathrm{kbps})$, the lower-band enhancement layer can be switched on. As seen in Figure 2-5, the lower-band enhancement layer increases the number of quantization levels available. The finer quantization allows it to produce results with less quantization noise. This provides 


\section{Tree Encoding in the ITU-T G.711.1 Speech Coder}

an increase in performance (Table 5-2), but at the cost of increased data rate. This performance increase is more noticeable in the case of the attenuated signal. In this scenario, when a reduced number of quantization levels are used, the enhancement layer reaps the full benefit of its increased quantization levels. Tree encoding does take a step towards reaching this performance level, but at the original data rate requirement of $64 \mathrm{kbps}$. Another advantage of tree encoding is that while the lowerband enhancement layer is not compatible with the legacy G.711 decoders, G.711.1 core layer with tree encoding is. Hence, increase in performance at the same data rate without the replacement of the already installed G.711 decoders can be achieved.

In a subjective test, for the signal without attenuation, at $64 \mathrm{kbps}$, the difference between the signals encoded with the different encoders is barely noticeable. Though, it can be said with certainty that the subjective quality of the signals encoded with the modified encoders is not less than that of the signal encoded with the original G.711.1 core layer encoder. For the attenuated signal, the modified encoders provide a better result. The speech is less broken, especially in the case of the tree encoder, as it has access to the future values while making the decision. As was the case with the PESQ scores, with the addition of the lower-band enhancement layer, at $80 \mathrm{kbps}$, the increase in subjective quality is even more still. Even though tree encoding does not reach this quality, it does have two advantages over the use of the lower-band enhancement layer. The increase in quality due to 


\section{Tree Encoding in the ITU-T G.711.1 Speech Coder}

tree encoding can be availed without any increase in data rate and with the already installed G.711 decoders.

\subsubsection{PERFORMANCE AS A FUNCTION OF M}

As stated earlier, $M$ defines the spread of the tree. More precisely it is the maximum number of nodes kept at the end of each decision making instance where the optimal output decision is made for a delayed input block. It was shown that $M$ is upper bounded, and depends on the size of the codebook and on the depth of the tree, or the artificial delay. Increase in $M$, within this upper bound, means more nodes are kept back after each decision making instance. Hence, more leaves are available when a new input signal is received, depending on the size of the sub optimal codebook.

The performance of the system as a function of $M$ for a signal attenuation of $-40 d B$ is plotted in Figure 5-1. Performance of G.711.1 core layer is provided for reference. The value of $L$ is kept constant at 6 except for the case when $M=1$. In that case, $L$ is equal to 1 as well, as described in an earlier section. The performance increases as the tree encoder is turned on, but it saturates very quickly. Subjectively there is not much difference between the different tree encoded signals as such a small difference cannot be detected. 


\section{Coder}

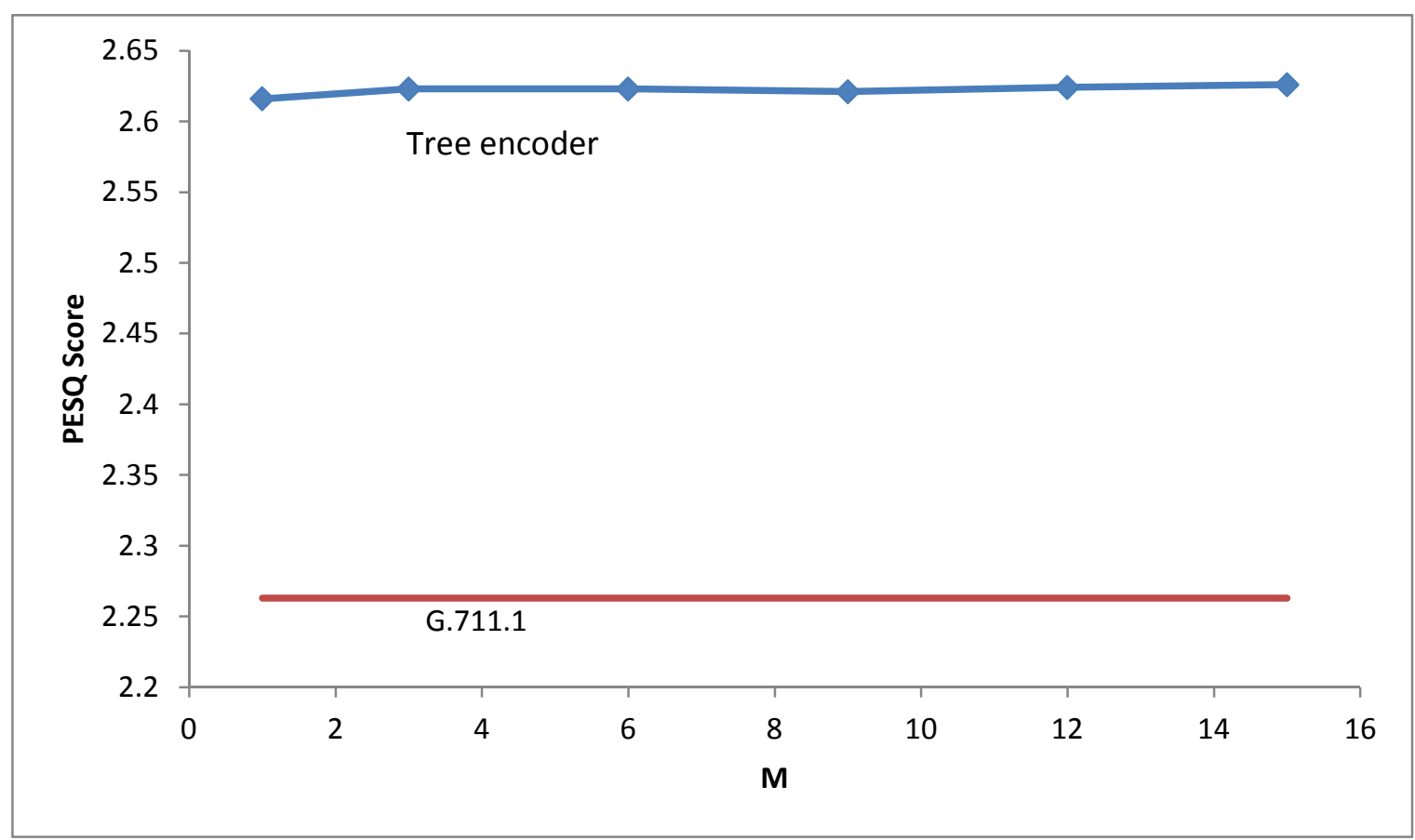

Figure 5-1 - PESQ score of tree encoding as a function of $M$, with $L=6$ at $-40 \mathrm{~dB}$.

For the first point $M=1$ and $L=1$. The performance of $G .711 .1$ core layer is provided for comparison.

In the case of a $\mu$-law quantizer, where the quantizer has a large range and large quantization intervals, there will be a lot of codewords which do not give satisfactory error results when used to approximate the input block. With a smaller $M$ these tend to get eliminated very quickly. Only the good approximations are kept. The benefit can be seen by the sudden increase in the performance of the encoder as compared to the original G.711.1 core layer encoder.

The saturation of the performance is because when $M$ increases many of the codewords with bad performance are kept. The tree encoder keeps on ignoring them because they do not give good results. The cumulative errors on these paths 


\section{Tree Encoding in the ITU-T G.711.1 Speech Coder}

keep on increasing. Even certain good approximations down the path cannot reduce the accumulated sum. Even though these are kept due to a large value of $M$ they are not chosen because there are other options available with better cumulative errors. Hence, the optimal path is more or less the same. Therefore, high performance can be achieved without taking $M$ to its upper bound. Once saturated, any increase in $M$ only results in increase of encoder complexity.

\subsubsection{PERFORMANCE AS A FUNCTION OF L}

The parameter $L$ defines the depth of the tree. More specifically it defines how many blocks of input data would be looked at before making the decision. Hence, it also defines the artificial delay that is created. The purpose of $L$ is that the quantization error be averaged out over a larger number of samples. Once $L>1$, multi-path tree encoding is realized. It also defines the upper bound on $M$, hence, the spread of the tree. This bound increases drastically with an increasing $L$ and, hence, soon loses its significance. 


\section{Coder}

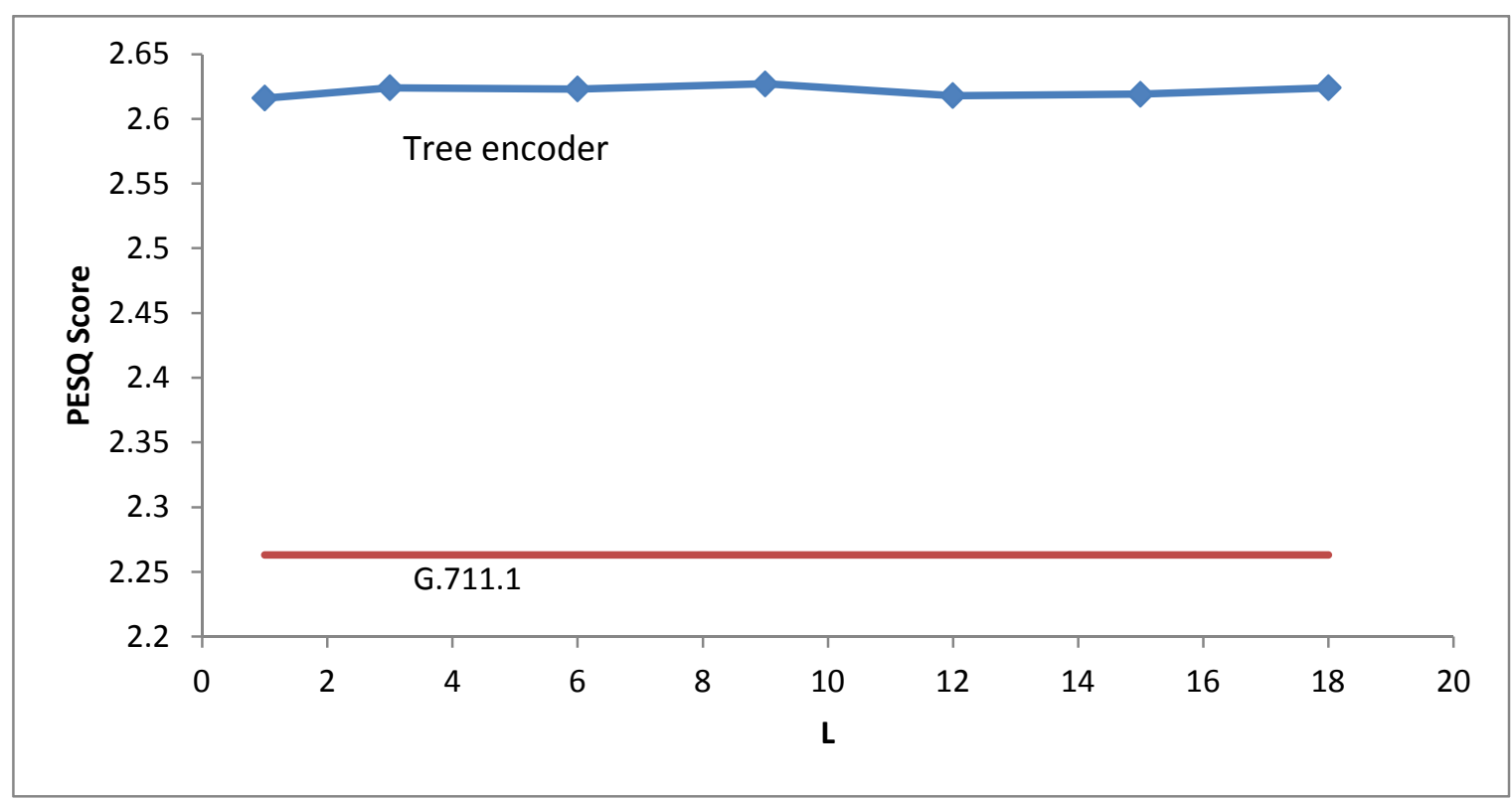

Figure 5-2 - PESQ score of tree encoding as a function of $L$, with $M=6$ at $-40 \mathrm{~dB}$.

For the first point $L=1$ and $M=1$. The performance of $G .711 .1$ core layer is provided for comparison.

Figure 5-2 shows the perceptual performance of the tree encoder as a function of $L$, at a signal attenuation of $-40 \mathrm{~dB}$. The PESQ score of G.711.1 core layer is provided as reference. For the case of $L=1, M$ is also equal to unity. As was the case with $M$ it is seen that there is a sudden increase in performance as the tree encoder is kicked in and then the performance saturates out. In the beginning the benefit is because the encoder can look at more values and it can take into consideration the effect on future values. After a limit the quantization error cannot be averaged out further more. Also, the noise feedback filter has a filter memory of 4 and the block size is 2 . With a small $L$ the effect on future samples due to filter memory is taken care of. Once $L$ gets large the benefit tends to decrease because the 

Coder

major effect of filter memory is only short term. Also, the large quantization intervals of $\mu$-law quantizer do not provide enough room in terms of selection of different codewords. A further increase in the tree depth only results in an increase of encoder complexity without any performance increase. 


\section{Chapter 6 CONCLUSION}

The use of delayed decision coding in ITU-T G.711.1 wideband extension standard has been studied in this thesis. The thesis started off by describing the core layer of the standard in Chapter 2. In Chapter 3 the similarities between vector quantized ADPCM and CELP coding were discussed. Similar structures for both were derived and a similar modification of inclusion of vector quantizer in G.711.1 core layer quantizer was proposed. To counter the effect of error propagation due to filter memory delayed decision coding was introduced in Chapter 4 . The $(M, L)-$ algorithm used to implement the tree encoder was described in detail. It was followed by Chapter 5 which contained the computer simulation and performance results.

The simulation results show that tree encoding gives better results than the G.711.1 core layer. The performance increase is limited by the $\mu$-law quantizer as the quantizer has a large range and large quantization intervals which restrict the increase in performance that can be achieved by a tree encoder. Also, due to the large number of quantization levels the codebook size is huge, limiting the block 


\section{Tree Encoding in the ITU-T G.711.1 Speech Coder}

size. Furthermore, the codebook had to be sub optimized to keep the complexity under check.

Further studies for this system are recommended. With the increase in hardware capability a larger codebook can be used instead of the sub optimized version. If sub optimization is the case then it can be improved by a better technique. One technique could be adaptive sub optimization where the selection of the neighbourhood of the input sample is different when in a different location of the quantizer range. The $\mu$-law quantizer has larger quantization intervals when the signal is large and smaller ones for low level signals. This could be put to use to have a larger neighbourhood in the case of low intensity signals and smaller neighbourhood for large amplitude signals. Larger input block sizes could be considered. 


\section{Tree Encoding in the ITU-T G.711.1 Speech 2010 Coder}

\section{REFERENCES}

[1] J.-H. Chen, "Novel Codec Structures for Noise Feedback Coding of Speech", Proc. IEEE Int. Conf. Acoustics, Speech, Signal Processing, pp. I-681-I-684, May 2006.

[2] ITU-T Recommendation G.711.1, Wideband embedded extension for G.711 pulse code modulation, March 2008.

[3] C. C. Chu, Tree Encoding of Speech Signals at Low Bit Rates, M.Eng. Thesis, Dept. Electrical \& Computer Engineering, McGill University, March 1986 (available on-line at www-mmsp.ece.mcgill.ca/Theses).

[4] J. Lapierre, R. Lefebvre, B. Bessette, V. Malenovsky and R. Salami, "Noise Shapping In An ITU-T G.711-Interoperable Embedded Codec", $16^{\text {th }}$ European Signal Processing Conference (EUSIPCO 2008), Lausanne, Switzerland, August 25-29, 2008.

[5] P. Kabal, The Equivalence of ADPCM and CELP Coding, Technical Report, Dept. Electrical \& Computer Engineering, McGill University, April 2010 (available on-line at www-mmsp.ece.mcgill.ca) 


\section{Tree Encoding in the ITU-T G.711.1 Speech Coder}

[6] V. Iyengar, A Low Delay 16 kbit/sec Coder for Speech Signals, M.Eng. Thesis, Electrical \& Computer Engineering, McGill University, Aug. 1987 (available on-line at www-mmsp.ece.mcgill.ca/MMSP/Theses).

[7] V. Iyengar and P. Kabal, "A Low Delay 16 kbits/sec Speech Coder", Proc., IEEE Int. Conf. Acoust., Speech, Signal Processing (New York, NY), pp. 243-246, April 1988.

[8] J. B. Anderson, J. B. Bodie, "Tree Encoding of Speech", IEEE Transactions on Information Theory, Vol. IT-21, No. 4, July 1975.

[9] A. W. Rix, J. G. Beerends, M. P. Hollier and A. P. Hekstra, "Perceptual Evaluation of Speech Quality (PESQ)-A New Method for Speech Quality Assessment of Telephone Networks and Codes", Proc. IEEE International Conference on Acoustic, Speech and Signal Processing, Salk Lake City, USA, 2001, pp. 749-752.

[10] ITU-T P.862, Perceptual evaluation of speech quality (PESQ): An objective method for end-to-end speech quality assessment of narrow-band telephone networks and speech codes, February 2001.

[11] J.-L. Garcia, C. Marro and B. Kövesi, "A PCM Coding Noise Reduction for ITU-T G.711.1", INTERSPEECH-2008, pp. 57-60.

[12] N. S. Jayant and P.Noll, Digital Coding of Waveforms, Prentice-Hall, 1984 (ISBN978-0-13-211913-9).

[13] Y. Hiwasaki, S. Sasaki, H. Ohmuro, T. Mori, J. Seong, M. S. Lee, B. Kövesi, S. Ragot, J.-L. Garcia, C. Marro, L. Miao, J. Xu, V. Malenovsky, J. Lapierre and R. Lefebvre, 


\section{Tree Encoding in the ITU-T G.711.1 Speech 2010 Coder}

“G.711.1: A Wideband Extension to ITU-T G.711", European Signal Processing Conf. (Lausanne, Switzerland), 4 pp. Aug. 2008

[14] J. Thyssen and J.-H. Chen, "Efficient VQ techniques and general noise shaping in noise feedback coding", INTERSPEECH-2006, paper 1254-Mon1FoP.5.

[15] S. Sasaki, T. Mori, Y. Hiwasaki and H. Ohmuro, Global Standard for Wideband Speech Coding: ITU-T G.711.1 (G.711 wideband extension), NTT Technical Review. 